\title{
QUANTUM COHOMOLOGY OF THE LAGRANGIAN GRASSMANNIAN
}

\author{
ANDREW KRESCH AND HARRY TAMVAKIS
}

\begin{abstract}
Let $V$ be a symplectic vector space and $L G$ be the Lagrangian Grassmannian which parametrizes maximal isotropic subspaces in $V$. We give a presentation for the (small) quantum cohomology ring $Q H^{*}(L G)$ and show that its multiplicative structure is determined by the ring of $\widetilde{Q}$-polynomials. We formulate a 'quantum Schubert calculus' which includes quantum Pieri and Giambelli formulas, as well as algorithms for computing the structure constants appearing in the quantum product of Schubert classes.
\end{abstract}

\section{INTRODUCTION}

The multiplicative structure of the quantum cohomology ring $Q H^{*}(\mathfrak{X})$ of a projective complex manifold $\mathfrak{X}$ encodes the enumerative geometry of rational curves in $\mathfrak{X}$, in the form of Gromov-Witten invariants. The ring $Q H^{*}(\mathfrak{X})$ is a deformation of the cohomology ring $H^{*}(\mathfrak{X})$ which first appeared in the work of string theorists (see e.g. [V], [W]). The exposition in [FP], following the work of Kontsevich and Manin KM], gives an algebro-geometric approach to this theory when $\mathfrak{X}$ is a homogeneous space $G / P$, where $G$ is a complex Lie group and $P$ a parabolic subgroup, which is our main interest here (see also [LT]). We will work throughout with the small quantum cohomology ring (terminology from [FP $\S 10]$ ).

The cohomology rings of homogeneous spaces $\mathfrak{X}=G / P$ have been studied extensively; see [Bo] BGG] D1 [D2. We would like to have an analogous description of the multiplicative structure of $Q H^{*}(\mathfrak{X})$, which we refer to as quantum Schubert calculus. As in the classical case, there are three main ingredients necessary for the latter theory: (i) a presentation of $Q H^{*}(\mathfrak{X})$ in terms of generators and relations, (ii) a quantum Giambelli formula which identifies the polynomials which represent the Schubert classes in this presentation, and (iii) algorithms for computing the structure constants in the multiplication table of $Q H^{*}(\mathfrak{X})$ (the latter include quantum Pieri rules).

Currently we have a fairly complete understanding these questions when $\mathfrak{X}=$ $S L_{n} / P$ is a partial flag variety of $S L_{n}(\mathbb{C})$. Since the theory is not functorial, a separate analysis must be done for the various parabolic subgroups $P$. For work (in the $S L_{n}$ case) on the quantum cohomology of (i) Grassmannians, see [W], [ST], $[\mathrm{Be}, \mathrm{BCF}$, (ii) complete flag varieties, see [GK], [C-F1, [FGP] and (iii) partial flag varieties, see $\mathrm{AS}$, K1, K2, C-F2, C].

In contrast to the $S L_{n}$ situation, much less is understood for the other families of Lie groups. For an arbitrary complex semisimple Lie group $G$, with Borel subgroup

Date: May 3, 2003.

2000 Mathematics Subject Classification. 14M15; 05E15.

Key words and phrases. Quantum cohomology, Quot schemes, Schubert calculus. 
$B$, Kim [K3] found the quantum $\mathcal{D}$-module structure for the flag variety $\mathfrak{X}=G / B$, and thus determined a presentation of $Q H^{*}(\mathfrak{X})$. To the authors' knowledge one still lacks a presentation of the quantum ring for the other parabolic subgroups and a quantum Giambelli formula to compute the Gromov-Witten invariants, if $G$ is not of type $A$. Our aim in this paper is to answer all three questions when the Lie group $G=S p_{2 n}$ is the symplectic group and $P=P_{n}$ is the maximal parabolic subgroup associated with a 'right end root' in the Dynkin diagram for the root system of type $C_{n}$. In this case $\mathfrak{X}=G / P$ is the Lagrangian Grassmannian described below. In a companion paper to this one KT2, we describe the corresponding story for the orthogonal groups.

All cohomology classes in this paper occur in even degrees. To avoid unnecessary factors of two, we adopt the following convention: a class $\alpha$ in the cohomology of a complex variety $\mathfrak{X}$ has degree $k$ when $\alpha$ lies in $H^{2 k}(\mathfrak{X}, \mathbb{Z})$.

Let $V$ be a complex vector space of dimension $2 n$, equipped with a symplectic form. The variety of Lagrangian (i.e., maximal isotropic) subspaces of $V$ is the Lagrangian Grassmannian $L G=L G(n, 2 n)=S p_{2 n} / P_{n}$. The integral cohomology ring $H^{*}(L G, \mathbb{Z})$ has a $\mathbb{Z}$-basis of Schubert classes $\sigma_{\lambda}$, one for each strict partition $\lambda=\left(\lambda_{1}>\lambda_{2}>\cdots>\lambda_{r}\right)$ with $\lambda_{1} \leqslant n$. Each $\sigma_{\lambda}$ is the class of a Schubert variety $\mathfrak{X}_{\lambda}$ of codimension $|\lambda|=\sum \lambda_{i}$ in $L G$. Of particular importance are the classes $\sigma_{i, j}$, determined by two Schubert conditions, and the special Schubert classes $\sigma_{i}=\sigma_{i, 0}$, defined by a single Schubert condition. This terminology is reviewed in 3.1

To describe the multiplicative structure of the classical and quantum cohomology ring of $L G$ we will use the $\widetilde{Q}$-polynomials of Pragacz and Ratajski $[\mathrm{PR}]$. These symmetric polynomials are modeled on Schur's $Q$-functions $[\underline{\mathrm{S}}$, and are the geometric analogues of Schur's $S$-functions in type $C$. Let $X_{k}=\left(x_{1}, \ldots, x_{k}\right)$ be a $k$-tuple of variables, let $X=X_{n}$, and for $i \geqslant 0$ let $\widetilde{Q}_{i}(X)$ be the $i$-th elementary symmetric polynomial $e_{i}(X)$. For any nonnegative integers $i, j$ with $i \geqslant j$, let

$$
\widetilde{Q}_{i, j}(X)=\widetilde{Q}_{i}(X) \widetilde{Q}_{j}(X)+2 \sum_{k=1}^{j}(-1)^{k} \widetilde{Q}_{i+k}(X) \widetilde{Q}_{j-k}(X),
$$

and for any partition $\lambda=\left(\lambda_{1} \geqslant \lambda_{2} \geqslant \cdots \geqslant \lambda_{r} \geqslant 0\right)$, define

$$
\widetilde{Q}_{\lambda}(X)=\operatorname{Pfaffian}\left[\widetilde{Q}_{\lambda_{i}, \lambda_{j}}(X)\right]_{1 \leqslant i<j \leqslant r},
$$

if $r$ is even; we may always assume this is the case by setting $\lambda_{r}=0$ if necessary. For each positive integer $n$ we denote by $\mathcal{D}_{n}$ the set of strict partitions $\lambda$ with $\lambda_{1} \leqslant n$.

Let $\Lambda_{n}$ denote the ring $\mathbb{Z}[X]^{S_{n}}$ of symmetric polynomials in $X$; as an abelian group, $\Lambda_{n}$ is spanned by the polynomials $\widetilde{Q}_{\lambda}(X)$ for all partitions $\lambda$. From the analysis in $\left[\mathrm{P}\right.$, Sect. 6] and $[\mathrm{PR}]$ we obtain that the map which sends $\widetilde{Q}_{\lambda}(X)$ to $\sigma_{\lambda}$ for all $\lambda \in \mathcal{D}_{n}$ extends to a surjective ring homomorphism $\phi: \Lambda_{n} \rightarrow H^{*}(L G, \mathbb{Z})$ with kernel generated by the relations $\widetilde{Q}_{i, i}(X)=0$ for $1 \leqslant i \leqslant n$; the morphism $\phi$ is evaluation on the Chern roots of the tautological rank $n$ quotient bundle over $L G$. From this statement one gets a presentation for the cohomology ring of $L G$; moreover, equations (11) and (2) become Giambelli-type formulas, expressing the Schubert classes in terms of the special ones.

The quantum cohomology $Q H^{*}(L G)$ of the Lagrangian Grassmannian $L G(n, 2 n)$ is an algebra over $\mathbb{Z}[q]$, where $q$ is a formal variable of degree $n+1$; one recovers the classical cohomology ring by setting $q=0$. For the $S L_{n}$-Grassmannian, Bertram 
Be proved the remarkable fact that the classical and quantum Giambelli are identical; note that this is in contrast to the other $S L_{n}$-flag varieties ( $\mathrm{FGP}$, [C-F2], C]). Strictly speaking, the quantum Giambelli formulas for $L G$ do not coincide with the classical ones. Let $X^{+}:=X_{n+1}$, and define $\widetilde{\Lambda}_{n+1}$ to be the subring of $\Lambda_{n+1}$ generated by the polynomials $\widetilde{Q}_{i}\left(X^{+}\right)$for $i \leqslant n$ together with the polynomial $2 \widetilde{Q}_{n+1}\left(X^{+}\right)$; the latter will play the role of $q$ in the quantum cohomology ring. Equations (11) and (2) imply that $\widetilde{Q}_{\lambda}\left(X^{+}\right) \in \widetilde{\Lambda}_{n+1}$ for all partitions $\lambda$ with $\lambda_{1} \leqslant n$.

Theorem 1. The map sending $\widetilde{Q}_{\lambda}\left(X^{+}\right)$to $\sigma_{\lambda}$ for all $\lambda \in \mathcal{D}_{n}$ and $2 \widetilde{Q}_{n+1}\left(X^{+}\right)$ to $q$ extends to a surjective ring homomorphism $\widetilde{\Lambda}_{n+1} \rightarrow Q H^{*}(L G)$ with kernel generated by the relations $\widetilde{Q}_{i, i}\left(X^{+}\right)=0$ for $1 \leqslant i \leqslant n$. The ring $Q H^{*}(L G)$ is presented as a quotient of the polynomial ring $\mathbb{Z}\left[\sigma_{1}, \ldots, \sigma_{n}, q\right]$ by the relations

$$
\sigma_{i}^{2}+2 \sum_{k=1}^{n-i}(-1)^{k} \sigma_{i+k} \sigma_{i-k}=(-1)^{n-i} \sigma_{2 i-n-1} q,
$$

where it is understood that $\sigma_{j}=0$ for $j<0$. The Schubert class $\sigma_{\lambda}$ in this presentation is given by the Giambelli formulas

$$
\sigma_{i, j}=\sigma_{i} \sigma_{j}+2 \sum_{k=1}^{n-i}(-1)^{k} \sigma_{i+k} \sigma_{j-k}+(-1)^{n+1-i} \sigma_{i+j-n-1} q
$$

for $i>j>0$, and

$$
\sigma_{\lambda}=\operatorname{Pfaffian}\left[\sigma_{\lambda_{i}, \lambda_{j}}\right]_{1 \leqslant i<j \leqslant r},
$$

where quantum multiplication is employed throughout. In other words, quantum Giambelli for $L G(n, 2 n)$ coincides with classical Giambelli for $L G(n+1,2 n+2)$, when the class $2 \sigma_{n+1}$ is identified with $q$.

Our proof of Theorem 1 is similar to $\mathrm{Be}$ in that we use the Lagrangian Quot scheme $L Q_{d}$ to compactify the moduli space of degree $d$ maps $\mathbb{P}^{1} \rightarrow L G$. However, unlike the Quot scheme in type $A$, the scheme $L Q_{d}$ is singular in general. More significantly, in [KT1] we showed that there is no direct analogue of the KempfLaksov formula $\mathrm{KL}$ for isotropic morphisms in type $C$. Thus the key intersectiontheoretic ingredient used by Bertram $\mathrm{Be}$ to prove quantum Giambelli in type $A$ is no longer available. Instead, we first show how to evaluate any three term Gromov-Witten invariant in degree 1 (i.e., one that counts lines on $L G$ satisfying three incidence conditions); this provides the quantum Giambelli formula (4) for the Schubert classes $\sigma_{i, j}$, as well as the ring presentation for $Q H^{*}(L G)(3.3)$. The Pfaffian formula (5) then follows from a relation in the Chow group of Lagrangian Quot schemes, which is proved using a careful analysis of the boundary of $L Q_{d}$ and some remarkable Pfaffian identities for certain symplectic Schubert polynomials (\$2.3). The latter objects were introduced in [PR] and represent the Schubert classes in the complete symplectic flag variety.

In $Q H^{*}(L G)$ there are formulas

$$
\sigma_{\lambda} \cdot \sigma_{\mu}=\sum e_{\lambda \mu}^{\nu}(n) \sigma_{\nu} q^{d}
$$

the sum over $d \geqslant 0$ and strict partitions $\nu$ with $|\nu|=|\lambda|+|\mu|-d(n+1)$. The quantum structure constant $e_{\lambda \mu}^{\nu}(n)$ is equal to a Gromov-Witten invariant $\left\langle\sigma_{\lambda}, \sigma_{\mu}, \sigma_{\nu^{\prime}}\right\rangle_{d}$, which is a non-negative integer. The integer $\left\langle\sigma_{\lambda}, \sigma_{\mu}, \sigma_{\nu^{\prime}}\right\rangle_{d}$ counts the number of 
rational curves of degree $d$ that meet three Schubert varieties $\mathfrak{X}_{\lambda}, \mathfrak{X}_{\mu}$ and $\mathfrak{X}_{\nu^{\prime}}$ in general position. Here $\nu^{\prime}$ is the dual partition of $\nu$, defined so that the classes $\sigma_{\nu}$ and $\sigma_{\nu^{\prime}}$ are Poincaré dual to each other in $H^{*}(L G, \mathbb{Z})$.

Using Theorem 1 we give formulas and algorithms to compute the quantum numbers $e_{\lambda \mu}^{\nu}(n)$; our approach was inspired by the second author's study [T] of Arakelov theory on Lagrangian Grassmannians. The resulting 'quantum Schubert calculus' is a natural extension of the classical formulas of Hiller and Boe $\mathrm{HB}$, Stembridge $\underline{\mathrm{St}}$ and Pragacz $\underline{\mathrm{P}}$. In particular we obtain a 'quantum Pieri rule' (Proposition 8) for multiplying by a special Schubert class in $Q H^{*}(L G)$, and some cases of a general 'quantum Littlewood-Richardson rule' in type $C$, which we obtain by relating some of the higher degree quantum structure constants with classical ones (Theorem 7 and Corollary [7).

This paper is organized as follows. In Section 2 we study the $\widetilde{Q}$-polynomials and symplectic Schubert polynomials, and prove the Pfaffian identities we need for the latter family. The Lagrangian and isotropic Grassmannians are introduced in Section 3] which includes our proof of the easier part (4) of the quantum Giambelli formula for $L G$. We complete the proof of Theorem 1 in Sections 4 and 5 by studying intersections on the Lagrangian Quot scheme $L Q_{d}$. Finally, in Section 6 we formulate a 'quantum Schubert calculus' for $L G$ which extends the classical one.

The authors thank Anders Buch and Bill Fulton for helpful correspondence. Both authors were supported in part by National Science Foundation post-doctoral research fellowships. We also wish to thank the Institut des Hautes Études Scientifiques for its hospitality during the final stages of this work.

\section{2. $\widetilde{Q}$-POLYNOMIALS AND SYMPlectic SChUbert POLYNOMIALS}

2.1. Basic definitions and properties. We begin by recalling some basic facts about partitions and their Young diagrams; the main reference is [M]. A partition is a sequence $\lambda=\left(\lambda_{1}, \lambda_{2}, \ldots, \lambda_{r}\right)$ of nonnegative integers in decreasing order; the length $\ell(\lambda)$ is the number of (nonzero) parts $\lambda_{i}$, and the weight $|\lambda|=\sum \lambda_{i}$. Set $\lambda_{k}=0$ for any $k>\ell(\lambda)$. We identify a partition with its associated Young diagram of boxes; the relation $\lambda \supset \mu$ is defined by the containment of diagrams. When $\lambda \supset \mu$ the set-theoretic difference is the skew diagram $\lambda / \mu$. A skew diagram $\alpha$ is a horizontal strip if it has at most one box in each column. Two boxes in $\alpha$ are connected if they share a vertex or an edge; this defines the connected components of $\alpha$.

Let $\mathcal{E}_{n}$ denote the set of all partitions $\lambda$ with $\lambda_{1} \leqslant n$. A partition is strict if all its parts are different. Let $\rho_{n}=(n, n-1, \ldots, 1)$ and recall that $\mathcal{D}_{n}$ denotes the set of strict partitions $\lambda$ with $\lambda \subset \rho_{n}$. We use the notation $\lambda \backslash \mu$ to denote the partition with parts given by the parts of $\lambda$ which are not parts of $\mu$.

Our references for the polynomials in this section are $[\mathrm{PR}]$ and $[\mathrm{LP}$; we will follow the notational conventions of [KT1 throughout most of the paper. Recall from the introduction the definitions of the polynomials $\widetilde{Q}_{\lambda}(X)$ and the ring $\Lambda_{n}$ of symmetric polynomials in $X=X_{n}$. These polynomials enjoy the following properties [PR, Sect. 4]:

(a) If $\lambda_{1}>n$, then $\widetilde{Q}_{\lambda}(X)=0$.

(b) The set $\left\{\widetilde{Q}_{\lambda}(X) \mid \lambda \in \mathcal{E}_{n}\right\}$ is a $\mathbb{Z}$-basis for $\Lambda_{n}$. 
(c) $\widetilde{Q}_{i, i}(X)=e_{i}\left(X_{1}^{2}, \ldots, X_{n}^{2}\right)$ for all $i$.

(d) $\widetilde{Q}_{n}(X) \widetilde{Q}_{\lambda}(X)=\widetilde{Q}_{(n, \lambda)}(X)$ for all $\lambda \in \mathcal{E}_{n}$.

(e) If $\lambda=\left(\lambda_{1}, \ldots, \lambda_{r}\right)$ and $\lambda^{+}=\lambda \cup(i, i)=\left(\lambda_{1}, \ldots, i, i, \ldots, \lambda_{r}\right)$ then

$$
\widetilde{Q}_{\lambda^{+}}(X)=\widetilde{Q}_{i, i}(X) \widetilde{Q}_{\lambda}(X) .
$$

Define a composition $\nu$ to be a sequence of nonnegative integers with finitely many nonzero parts; we set $|\nu|=\sum \nu_{i}$. The $\widetilde{Q}$-polynomials make sense when indexed by a composition $\nu$ (in fact, the index can be an arbitrary finite-length sequence of integers, following [LP, Rmk. 5.3] — we will not need this here). For any composition $\nu$ the polynomial $\widetilde{Q}_{\nu}(X)$ is equal to $\pm \widetilde{Q}_{\lambda}(X)$, where $\lambda$ is the partition obtained from $\nu$ by reordering the parts of $\nu$. The sign is determined by the following rule:

$$
\widetilde{Q}_{(\ldots, i, j, \ldots)}(X)=-\widetilde{Q}_{(\ldots, j, i, \ldots)}(X)
$$

for any two adjacent indices $i, j$ with $i \neq j$. In other words, for any composition $\nu$ we have $\widetilde{Q}_{\nu}(X)=\operatorname{sgn}(\sigma) \widetilde{Q}_{\lambda}(X)$, where $\sigma \in S_{\infty}$ is the permutation of minimal length such that $\nu_{\sigma(k)}=\lambda_{k}$ for all $k$. The polynomials defined in this way satisfy Pfaffian relations such as

$$
\widetilde{Q}_{\nu}(X)=\sum_{j=1}^{r-1}(-1)^{j-1} \widetilde{Q}_{\nu_{j}, \nu_{r}}(X) \cdot \widetilde{Q}_{\nu \backslash\left\{\nu_{j}, \nu_{r}\right\}}(X),
$$

where $r$ is an even number such that $\nu_{i}=0$ for $i>r$; this follows e.g. from the Laplace-type expansion for Pfaffians displayed in [FPr, (D.1)]. Using this convention, we have the following extension of $[\mathrm{PR}$ Prop. 4.1]:

Proposition 1. For any partition $\lambda$ (not necessarily strict) we have

$$
\widetilde{Q}_{\lambda}(X)=\sum_{k=0}^{\ell(\lambda)} x_{1}^{k} \sum_{\mu} \widetilde{Q}_{\mu}\left(X^{\prime}\right),
$$

where the inner sum is over all compositions $\mu$ such that $|\lambda|-|\mu|=k$ and $\lambda_{i}-\mu_{i} \in$ $\{0,1\}$ for each $i$, while $X^{\prime}=\left(x_{2}, \ldots, x_{n}\right)$.

Proof. One notices the proof of [PR Prop. 4.1] carries over to this more general situation. In particular we have the relation

$$
\widetilde{Q}_{a, b}(X)=\widetilde{Q}_{a, b}\left(X^{\prime}\right)+x_{1}\left(\widetilde{Q}_{a-1, b}\left(X^{\prime}\right)+\widetilde{Q}_{a, b-1}\left(X^{\prime}\right)\right)+x_{1}^{2} \widetilde{Q}_{a-1, b-1}\left(X^{\prime}\right),
$$

which is true for any $a \geqslant b$. The rest of the argument is the same.

Remark. Proposition 10 fails when $\lambda$ is a composition; in fact (8) shows that it is false even in the case of two part compositions.

2.2. Symplectic Schubert polynomials. We denote the elements of the Weyl group $W_{n}$ for the root system $C_{n}$ as barred permutations, following [KT1, §1.1]. $W_{n}$ is generated by the elements $s_{0}, \ldots, s_{n-1}$ : for $i>0, s_{i}$ is the transposition interchanging $i$ and $i+1$, and we define $s_{0}$ by

$$
\left(u_{1}, u_{2}, \ldots, u_{n}\right) s_{0}=\left(\bar{u}_{1}, u_{2}, \ldots, u_{n}\right) .
$$

For each $\lambda \in \mathcal{D}_{n}$ of length $\ell$, with $k=n-\ell$ and $\lambda^{\prime}=\rho_{n} \backslash \lambda$, the barred permutation

$$
w_{\lambda}=\left(\bar{\lambda}_{1}, \ldots, \bar{\lambda}_{\ell}, \lambda_{k}^{\prime}, \ldots, \lambda_{1}^{\prime}\right)
$$


is the maximal Grassmannian element of $W_{n}$ corresponding to $\lambda$. We also define the elements

$$
w_{\lambda}^{\prime}=w_{\lambda} s_{0} \quad \text { and } \quad w_{\lambda}^{\prime \prime}=w_{\lambda} s_{0} s_{1} s_{0} .
$$

The group $W_{n}$ acts on the polynomial ring $\mathbb{Z}[X]$; in particular the transposition $s_{1}$ interchanges $x_{1}$ and $x_{2}$, while $s_{0}$ replaces $x_{1}$ by $-x_{1}$ (all other variables remain fixed). We require the divided difference operators $\partial_{0}, \partial_{1}^{\prime}: \mathbb{Z}[X] \rightarrow \mathbb{Z}[X]$, defined by

$$
\partial_{0}(f)=\left(f-s_{0} f\right) /\left(2 x_{1}\right) \quad \text { and } \quad \partial_{1}^{\prime}(f)=\left(f-s_{1} f\right) /\left(x_{2}-x_{1}\right) .
$$

Following $[\mathrm{PR}]$ and $\left[\mathrm{LP}\right.$, for each $w \in W_{n}$ there is a symplectic Schubert polynomial $\mathfrak{C}_{w}(X) \in \mathbb{Z}[X]$, which represents the Schubert class associated to $w$ in the cohomology ring of $S p_{2 n} / B$. According to [LP Thm. A.6], for each $\lambda \in \mathcal{D}_{n}$, the polynomial $\mathfrak{C}_{\lambda}(X):=\mathfrak{C}_{w_{\lambda}}(X)$ is equal to the $\widetilde{Q}$-polynomial $\widetilde{Q}_{\lambda}(X)$. Let us define

$$
\mathfrak{C}_{\lambda}^{\prime \prime}(X)=\mathfrak{C}_{w_{\lambda}^{\prime}}(X) \quad \text { and } \quad \mathfrak{C}_{\lambda}^{\prime \prime}(X)=\mathfrak{C}_{w_{\lambda}^{\prime \prime}}(X) ;
$$

it follows from (9) that

$$
\mathfrak{C}_{\lambda}^{\prime}(X)=\partial_{0}\left(\widetilde{Q}_{\lambda}(X)\right) \quad \text { and } \quad \mathfrak{C}_{\lambda}^{\prime \prime}(X)=\partial_{0} \partial_{1}^{\prime} \partial_{0}\left(\widetilde{Q}_{\lambda}(X)\right)
$$

(valid for $\ell(\lambda) \geqslant 1$ and $\ell(\lambda) \geqslant 2$, respectively).

2.3. Pfaffian identities. We next study certain identities for symplectic Schubert polynomials which are needed in our proof of the quantum Giambelli formula for $L G(n, 2 n)$. We see from (7) and (10) that for each strict partition $\lambda \in \mathcal{D}_{n}$,

$$
\mathfrak{C}_{\lambda}^{\prime}(X)=\sum_{\substack{k=0 \\ k \text { odd }}}^{\ell(\lambda)} x_{1}^{k-1} \sum_{\mu} \widetilde{Q}_{\mu}\left(X^{\prime}\right)
$$

with the inner sum over all partitions $\mu$ such that $|\lambda|-|\mu|=k$ and $\lambda_{i}-\mu_{i} \in\{0,1\}$ for each $i$. For example, we have

$$
\begin{aligned}
\mathfrak{C}_{a, b}^{\prime}(X) & =\widetilde{Q}_{a-1, b}\left(X^{\prime}\right)+\widetilde{Q}_{a, b-1}\left(X^{\prime}\right) \\
& =\widetilde{Q}_{a-1}\left(X^{\prime}\right) \widetilde{Q}_{b}\left(X^{\prime}\right)-\widetilde{Q}_{a}\left(X^{\prime}\right) \widetilde{Q}_{b-1}\left(X^{\prime}\right)
\end{aligned}
$$

for all $a, b$ with $a>b \geqslant 0$. Our aim is to prove the two Theorems that follow.

Theorem 2. Fix $\lambda \in \mathcal{D}_{n}$ of length $\ell \geqslant 3$, and set $r=2\lfloor(\ell+1) / 2\rfloor$. Then

$$
\sum_{j=1}^{r-1}(-1)^{j-1} \mathfrak{C}_{\lambda_{j}, \lambda_{r}}^{\prime}(X) \mathfrak{C}_{\lambda \backslash\left\{\lambda_{j}, \lambda_{r}\right\}}^{\prime}(X)=0 .
$$

Proof. Our argument can be conveniently expressed using only the partitions which index the polynomials involved; we therefore begin by defining an algebra with formal variables which represent these indices. Let $\mathcal{A}$ be a commutative $\mathbb{Z}$-algebra generated by symbols $\left(a_{1}, a_{2}, \ldots\right)$, where the entries $a_{i}$ are barred integers. The symbol $\left(a_{1}, a_{2}, \ldots\right)$ corresponds to the polynomial $\widetilde{Q}_{\rho}\left(X^{\prime}\right)$, where $\rho$ is the composition with $\rho_{i}$ equal to $a_{i}$, when $a_{i}$ is unbarred, and $a_{i}-1$ when $a_{i}$ is barred (in the proof, this is only used for partitions $\rho$ ). We identify $(a, 0)$ with $(a)$. 
Let $\mu$ be a barred partition, that is, a partition in which bars have been added to some of the entries; assume further that the underlying partition is strict. For $\ell(\mu) \geqslant 3$, we impose the Pfaffian relation

$$
(\mu)=\sum_{j=1}^{r-1}(-1)^{j-1}\left(\mu_{j}, \mu_{r}\right) \cdot\left(\mu \backslash\left\{\mu_{j}, \mu_{r}\right\}\right)
$$

(this corresponds to (6) for $\nu=\mu$ ). Iterating this relation gives

$$
(\mu)=\sum \epsilon(\mu, \nu)\left(\nu_{1}, \nu_{2}\right) \cdots\left(\nu_{r-1}, \nu_{r}\right),
$$

where the sum is over all $(r-1)(r-3) \cdots(1)$ ways to write the set $\left\{\mu_{1}, \ldots, \mu_{r}\right\}$ as a union of pairs $\left\{\nu_{1}, \nu_{2}\right\} \cup \cdots \cup\left\{\nu_{r-1}, \nu_{r}\right\}$, and where $\epsilon(\mu, \nu)$ is the sign of the permutation that takes $\left(\mu_{1}, \ldots, \mu_{r}\right)$ into $\left(\nu_{1}, \ldots, \nu_{r}\right)$; we adopt the convention that $\nu_{2 i-1} \geqslant \nu_{2 i}$.

We define the square bracket symbols $[\mu]$, where $\mu$ is a strict unbarred partition, as follows: $[a]=(\bar{a}),[a, b]=(\bar{a}, b)+(a, \bar{b})$, and generally

$$
[\mu]=\sum_{j=1}^{r-1}(-1)^{j-1}\left[\mu_{j}, \mu_{r}\right] \cdot\left[\mu \backslash\left\{\mu_{j}, \mu_{r}\right\}\right]
$$

Finally we impose the following relation in our algebra:

$$
[a, b]=(\bar{a})(b)-(a)(\bar{b})
$$

this corresponds to (12).

Lemma 1. For each $\mu \in \mathcal{D}_{n}$ with $\ell(\mu) \geqslant 3$, we have $[\mu]=0$ in $\mathcal{A}$.

Proof. When $\ell(\mu) \in\{3,4\}$ the identity is easy to check using (17). For $\mu$ of larger length it follows from the Laplace-type expansion for Pfaffians displayed in (16).

For a partition $\nu$ and integer $k$, we define $B(\nu, k)$ to be the set of all barred partitions $\mu$ which are obtained from $\nu$ by adding bars over $k$ distinct entries. Now, using equations (11) and (12) we see that (13) corresponds to the following identity, which must hold for each odd $k>0$ :

$$
\sum_{j=1}^{r-1}(-1)^{j-1}\left[\lambda_{j}, \lambda_{r}\right] \cdot \sum_{\mu \in B\left(\lambda \backslash\left\{\lambda_{j}, \lambda_{r}\right\}, k\right)}(\mu)=0 .
$$

To prove (18), we work as follows. Call a pair $\left[\nu_{2 i-1}, \nu_{2 i}\right]$ in square brackets distinguished; pairs which are not distinguished are called normal. A part of $\lambda$ is distinguished if it belongs to a distinguished pair. We say that a pair $\left(\nu_{2 i-1}, \nu_{2 i}\right)$ is odd if exactly one of the parts $\nu_{2 i-1}$ and $\nu_{2 i}$ is barred. In the sequel all brackets $\{$,$\} will be either round (,$,$) or square [$,$] , so all expressions are elements of the$ algebra $\mathcal{A}$.

We define a matching of a barred partition $\mu$ to be an expression of the set $\left\{\mu_{1}, \mu_{2}, \ldots, \mu_{r}\right\}$ as a union of pairs $\left\{\nu_{1}, \nu_{2}\right\} \cup \cdots \cup\left\{\nu_{r-1}, \nu_{r}\right\}$ as above, together with a specification of each $\left\{\nu_{2 i-1}, \nu_{2 i}\right\}$ as distinguished or normal. Fix an odd integer $k$ with $0<k<r-1$. The first claim is that the left hand side $S$ of the sum (18) satisfies

$$
S=\sum_{\mu} \sum_{\nu} \epsilon(\lambda, \nu)\left\{\nu_{1}, \nu_{2}\right\} \cdots\left\{\nu_{r-1}, \nu_{r}\right\}
$$


where (i) the outer sum is over $\mu \in B(\lambda, k)$ with the part equal to $\lambda_{r}$ unbarred, (ii) the inner sum is over all matchings $\nu$ of $\mu$ with a unique distinguished pair, which contains $\lambda_{r}$ (iii) the sign $\epsilon(\lambda, \nu)$ is defined as above, by ignoring the bars.

Let $t(\mu, \nu)$ be the total number of odd pairs in the summand

$$
\epsilon(\lambda, \nu)\left\{\nu_{1}, \nu_{2}\right\} \cdots\left\{\nu_{r-1}, \nu_{r}\right\}
$$

of $S$; note that $t(\mu, \nu)>0$. Observe, for each $t$, that the set of summands with $t(\mu, \nu)=t$ can be partitioned into subsets of size $2^{t}$ : elements in a single subset $J$ differ only by moving some of the bars within the odd pairs. The sum of the elements in $J$ is equal to a single expression $\epsilon(\lambda, \nu)\left\{\nu_{1}, \nu_{2}\right\} \cdots\left\{\nu_{r-1}, \nu_{r}\right\}$ with $t$ fewer barred parts, $t+1$ distinguished pairs and no odd pairs.

Working in this way we may eliminate all odd pairs from the sum $S$. Hence $S=S^{\prime}$, where

$$
S^{\prime}=\sum_{\mu}^{\sim} \sum_{\nu}^{\sim} \epsilon(\lambda, \nu)\left\{\nu_{1}, \nu_{2}\right\} \cdots\left\{\nu_{r-1}, \nu_{r}\right\}
$$

here (i) the outer sum is over all barred partitions $\mu$ with parts obtained by adding an even number, less than $k$, of bars to the parts of $\lambda$ (ii) the inner sum is over all matchings $\nu$ of $\mu$ such that $\lambda_{r}$ is distinguished and the number of barred parts plus the number of distinguished pairs equals $k+1$, (iii) the sign $\epsilon(\lambda, \nu)$ is defined by ignoring the bars, as before.

Observe next that $S^{\prime}$ splits into subsums $S^{\prime \prime}$; each $S^{\prime \prime}$ is the sum of all summands in $S^{\prime}$ with a given set of distinguished parts and normal pairs. It is clear that, up to a sign depending on $S^{\prime \prime}$, the remainder after factoring out the normal pairs from $S^{\prime \prime}$ is a Pfaffian sum like (15) which involves only distinguished pairs. Now Lemma 1 implies that $S^{\prime \prime}=0$; we deduce that $S^{\prime}$, and hence $S$, vanishes.

Theorem 3. For every $\lambda \in \mathcal{D}_{n}$ of even length $\ell \geqslant 4$ we have

$$
\sum_{j=1}^{\ell-1}(-1)^{j-1} \mathfrak{C}_{\lambda_{j}, \lambda_{\ell}}^{\prime \prime}(X) \mathfrak{C}_{\lambda \backslash\left\{\lambda_{j}, \lambda_{\ell}\right\}}^{\prime \prime}(X)=0 .
$$

Proof. Using (8) one computes that for $a>b>0$,

$$
\begin{aligned}
\partial_{0} \partial_{1}^{\prime} \partial_{0}\left(\widetilde{Q}_{a, b}(X)\right) & =\widetilde{Q}_{a-2, b-1}\left(X^{\prime \prime}\right)+\widetilde{Q}_{a-1, b-2}\left(X^{\prime \prime}\right) \\
& =\widetilde{Q}_{a-2}\left(X^{\prime \prime}\right) \widetilde{Q}_{b-1}\left(X^{\prime \prime}\right)-\widetilde{Q}_{a-1}\left(X^{\prime \prime}\right) \widetilde{Q}_{b-2}\left(X^{\prime \prime}\right),
\end{aligned}
$$

where $X^{\prime \prime}=\left(x_{3}, \ldots, x_{n}\right)$. We deduce that

$$
\operatorname{Pfaffian}\left[\mathfrak{C}_{\lambda_{i}, \lambda_{j}}^{\prime \prime}(X)\right]_{1 \leqslant i<j \leqslant \ell}=0
$$

for any partition $\lambda$ of even length $\ell \geqslant 4$, by arguing as in Lemma 1 The proof of the general case of (21) will require significantly more work.

For each $r$ and $s$ with $r \geqslant s \geqslant 0$, let $m_{r, s}\left(x_{1}, x_{2}\right)$ denote the monomial symmetric function in $x_{1}$ and $x_{2}$. In other words, $m_{r, s}\left(x_{1}, x_{2}\right)=x_{1}^{r} x_{2}^{s}+x_{1}^{s} x_{2}^{r}$ if $r \neq s$ and $m_{r, s}\left(x_{1}, x_{2}\right)=x_{1}^{r} x_{2}^{r}$ if $r=s$.

Proposition 2. For any strict partition $\lambda$ of even length $\ell>0$ we have

$$
\mathfrak{C}_{\lambda}^{\prime \prime}(X)=\sum_{\substack{0 \leqslant s \leqslant r<\ell \\
r, s \text { even }}} m_{r, s}\left(x_{1}, x_{2}\right) \sum_{\substack{a+2 b=r+s+3 \\
a, b \geqslant 0}}\left(\begin{array}{c}
a-1 \\
s+1-b
\end{array}\right) \sum_{\nu \in C(\lambda, a, b)} \widetilde{Q}_{\nu}\left(X^{\prime \prime}\right),
$$


where $C(\lambda, a, b)$ is defined as the set of compositions $\nu$ with $\lambda_{i}-\nu_{i} \in\{0,1,2\}$ for all $i$ and $\lambda_{i}-\nu_{i}=1$ (resp. $\lambda_{i}-\nu_{i}=2$ ) for exactly a (resp. b) values of $i$.

Proof. Use (10) and (11) to compute

$$
\mathfrak{C}_{\lambda}^{\prime \prime}(X)=\partial_{0} \partial_{1}^{\prime}\left(\sum_{\substack{j=0 \\ j \text { even }}}^{\ell-2} x_{1}^{j} \sum_{\mu \in P(\lambda, j+1)} \widetilde{Q}_{\mu}\left(X^{\prime}\right)\right),
$$

where $P(\lambda, j+1)$ is the set of compositions $\mu$ with $|\lambda|-|\mu|=j+1$ and $\lambda_{i}-\mu_{i} \in\{0,1\}$ for each $i$. Note also that for any $j, k \geqslant 0$ we have

$$
\partial_{1}\left(x_{1}^{j} x_{2}^{k}\right)=\operatorname{sgn}(j-k) \sum_{\substack{c+d=j+k-1 \\ c, d \geqslant \min \{j, k\}}} x_{1}^{c} x_{2}^{d} .
$$

For each strict partition $\lambda$ and $j, k \geqslant 0$, let $P(\lambda, j, k)$ be the set of pairs of compositions $(\mu, \nu)$ such that $\mu \in P(\lambda, j)$ and $\nu \in P(\mu, k)$. Now apply (17) and (24) in (23) to get

$$
\begin{aligned}
& \mathfrak{C}_{\lambda}^{\prime \prime}(X)=\sum_{j \text { even }} \partial_{0} \partial_{1}^{\prime}\left(x_{1}^{j} x_{2}^{k}\right) \sum_{(\mu, \nu) \in P(\lambda, j+1, k)} \widetilde{Q}_{\nu}\left(X^{\prime \prime}\right) \\
& =\sum_{j \text { even }} \operatorname{sgn}(k-j) \sum_{\substack{c+d=j+k-1 \\
c, d \geqslant \min \{j, k\} \\
c \text { odd }}} x_{1}^{c-1} x_{2}^{d} \sum_{(\mu, \nu) \in P(\lambda, j+1, k)} \widetilde{Q}_{\nu}\left(X^{\prime \prime}\right) .
\end{aligned}
$$

It is useful to observe that $\mathfrak{C}_{\lambda}^{\prime \prime}(X)$ is a polynomial in $R\left[x_{1}^{2}, x_{2}^{2}\right]^{S_{2}}$, where $R=$ $\mathbb{Z}\left[x_{3}, \ldots, x_{n}\right]^{S_{n-2}}$. This follows because

$$
\partial_{0}\left(\mathfrak{C}_{\lambda}^{\prime \prime}(X)\right)=\partial_{1}\left(\mathfrak{C}_{\lambda}^{\prime \prime}(X)\right)=0
$$

(for the latter, use the fact that $\partial_{1} \partial_{0} \partial_{1} \partial_{0}=\partial_{0} \partial_{1} \partial_{0} \partial_{1}$ and $\partial_{1}\left(\widetilde{Q}_{\lambda}(X)\right)=0$ ). Using this to cancel all irrelevant terms in the previous equality gives

$$
\mathfrak{C}_{\lambda}^{\prime \prime}(X)=\sum_{\substack{0 \leqslant s \leqslant r<\ell \\ r, s \text { even }}} m_{r, s}\left(x_{1}, x_{2}\right) \sum_{\substack{j, k \text { even } \\ j+k=r+s+2 \\ \min \{j, k\} \leqslant r, s}} \sum_{(\mu, \nu) \in P(\lambda, j+1, k)} \operatorname{sgn}(k-j) \widetilde{Q}_{\nu}\left(X^{\prime \prime}\right) .
$$

Consider the terms occuring in the inner sum $\sum \sum$ of 25] which involve a fixed $\nu \in C(\lambda, a, b)$, for some $a, b \geqslant 0$ such that $a+2 b=r+s+3$. Observe that the coefficient of any such term depends only on $(r, s, a, b)$ and can be expressed as

$$
\sum_{\substack{j, k \text { even } \\
j+k=r+s+2 \\
\min \{j, k\} \leqslant r, s}} \operatorname{sgn}(k-j)\left(\begin{array}{c}
a \\
k-b
\end{array}\right) .
$$

Now (26) can be rearranged as an alternating sum of binomial coefficients, which in turn simplifies to the single binomial coefficient displayed in (22).

For any $r$ and $s$, both even, the coefficient of $m_{r, s}\left(x_{1}, x_{2}\right)$ in the left-hand side of (21), expanded as a polynomial in $R\left[x_{1}^{2}, x_{2}^{2}\right]^{S_{2}}$, is

$$
\sum_{j=1}^{\ell-1}(-1)^{j-1} \mathfrak{C}_{\lambda_{j}, \lambda_{\ell}}^{\prime \prime}(X) \sum_{\substack{a+2 b=r+s+3 \\
a, b \geqslant 0}}\left(\begin{array}{c}
a-1 \\
s+1-b
\end{array}\right) \sum_{\nu \in C\left(\lambda \backslash\left\{\lambda_{j}, \lambda_{\ell}\right\}, a, b\right)} \widetilde{Q}_{\nu}\left(X^{\prime \prime}\right) .
$$


Hence, to prove Theorem 3 it suffices to show that the expression (27) vanishes for any strict partition $\lambda$ of even length $\ell \geqslant 4$ and any $r, s \geqslant 0$, both even.

To accomplish this, we form an algebra $\mathcal{B}$ as in the proof of Theorem 2 except that the symbols $\left(a_{1}, a_{2}, \ldots\right)$ are now sequences of integers which can each have up to two bars. This time the symbol $\left(a_{1}, a_{2}, \ldots\right)$ corresponds to the polynomial $\widetilde{Q}_{\nu}\left(X^{\prime \prime}\right)$, where $\nu$ is the composition with $\nu_{i}$ equal to the integer $a_{i}$ minus the number of bars over $a_{i}$.

As before, we impose the Pfaffian relations (14) in $\mathcal{B}$. Define also $[a, b]=(\bar{a}, b)+$ $(a, \bar{b})$, where $a$ and $b$ are integers, each with up to one bar. More generally, define $[\mu]$ for any barred partition $\mu$, as in (16). We also impose the relations

$$
[a, b]=(\bar{a})(b)-(a)(\bar{b})
$$

for integers $a, b$, with up to one bar each. As in the proof of Theorem 2 we have

Lemma 2. Let $\mu$ be a strict partition of even length at least 4 , such that either $\mu$ is unbarred or every part of $\mu$ has a bar. Then we have $[\mu]=0$ in $\mathcal{B}$.

The vanishing of (27) corresponds to the following identity in $\mathcal{B}$ :

$$
\sum_{\substack{a+2 b=r+s+3 \\
a, b \geqslant 0}}\left(\begin{array}{c}
a-1 \\
s+1-b
\end{array}\right) \sum_{j=1}^{\ell-1}(-1)^{j-1}\left[\bar{\lambda}_{j}, \bar{\lambda}_{\ell}\right] \sum_{\nu \in C\left(\lambda \backslash\left\{\lambda_{j}, \lambda_{\ell}\right\}, a, b\right)}(\nu)=0 .
$$

For each $a$ and $b$, denote by $W_{a, b}$ the contents of the inner two sums in (28). By applying the Pfaffian expansion to the terms $(\nu)$ in $W_{a, b}$, we can write

$$
W_{a, b}=\sum_{\rho} \epsilon(\lambda, \rho)\left\{\rho_{1}, \rho_{2}\right\} \cdots\left\{\rho_{\ell-1}, \rho_{\ell}\right\}
$$

The summands in $W_{a, b}$ are of three types: first, those that contain a pair $\left(\rho_{2 i-1}, \rho_{2 i}\right)$ with a total of three bars; second, those not of the first type which contain at least two pairs, each with exactly one bar; third, the remaining summands. Let $R_{a, b}$, $S_{a, b}$ and $T_{a, b}$ denote the sum of the summands of, respectively, the first, second and third types.

Arguing as in the proof of Theorem 2 and using Lemma 2 we see that $R_{a, b}=$ $S_{a, b}=0$, for all $a, b$. However, it is not true that $T_{a, b}$ is always zero. Observe that $T_{a, b}$ splits as a sum $\sum_{g} T_{a, b}^{g}$ indexed by the number $g$ of pairs in each summand which contain 4 bars. We will show that

$$
\sum_{\substack{a+2 b=r+s+3 \\
a, b \geqslant 0}}\left(\begin{array}{c}
a-1 \\
s+1-b
\end{array}\right) T_{a, b}^{g}=0
$$

for each $g$. In the following we give the argument when $g=0$, and afterwards we describe the small modifications for the proof of the general case.

Assume that $r \geqslant s$ and introduce

$$
u=(r-s) / 2 \quad \text { and } \quad v=(r+s) / 2 .
$$

We recursively define a sequence of coefficients: Put $e_{u}=1$ and

$$
e_{m}=\left(\begin{array}{c}
2 m \\
m-u
\end{array}\right)-\frac{2 m}{v+2-m} e_{m-1}
$$

for $m=u+1, \ldots, v+1$.

Lemma 3. We have $e_{v+1}=0$. 
Proof. For any integer $p \geqslant 0$ we have the combinatorial identity

$$
\sum_{k}(-1)^{k} 2^{-k}\left(\begin{array}{l}
p \\
k
\end{array}\right)\left(\begin{array}{c}
2 k \\
k-q
\end{array}\right)= \begin{cases}(-1)^{q} 2^{-p}\left(\begin{array}{c}
p \\
(p+q) / 2
\end{array}\right) & \text { if } p+q \text { is even } \\
0 & \text { if } p+q \text { is odd }\end{cases}
$$

This is proved, e.g., by showing the left-hand side $L(p, q)$ satisfies the recursion $2 L(p, q)+L(p-1, q-1)+L(p-1, q+1)=0$ and inducting on $p$. When $q=0$, (30) reduces to an identity attributed to Dawson in [R, p. 71]. The assertion $e_{v+1}=0$ follows from the case $(p, q)=(v+1, u)$ of (30).

We deduce from Lemma 3 that

$$
\begin{gathered}
\sum_{\substack{a+2 b=r+s+3 \\
a, b \geqslant 0}}\left(\begin{array}{c}
a-1 \\
s+1-b
\end{array}\right) T_{a, b}^{0}=\sum_{m=u}^{v+1}\left(\begin{array}{c}
2 m \\
m-u
\end{array}\right) T_{2 m+1, v+1-m}^{0} \\
=\sum_{m=u+1}^{v+1} e_{m-1}\left(T_{2 m-1, v+2-m}^{0}+\frac{2 m}{v+2-m} T_{2 m+1, v+1-m}^{0}\right) .
\end{gathered}
$$

Lemma 4. We have

$$
(v+2-m) T_{2 m-1, v+2-m}^{0}+2 m T_{2 m+1, v+1-m}^{0}=0
$$

for every integer $m$ with $u+1 \leqslant m \leqslant v+1$.

Proof. We have that

$$
T_{a, b}^{0}=\sum_{\mu \in C(\lambda, a+2, b)} \sum_{\nu} \epsilon(\lambda, \nu)\left\{\nu_{1}, \nu_{2}\right\} \cdots\left\{\nu_{\ell-1}, \nu_{\ell}\right\}
$$

where the inner sum is over all matchings $\nu$ of $\mu$ with (i) a unique distinguished pair, which contains $\lambda_{\ell}$ and has bars on both entries, (ii) a unique pair which contains only one bar, and (iii) no pairs which contain 3 or 4 bars.

Now,

$$
(v+2-m) T_{2 m-1, v+2-m}^{0}=\sum_{\mu \in C(\lambda, 2 m+1, v+2-m)} \sum_{(\nu, i)} \epsilon(\lambda, \nu)\left\{\nu_{1}, \nu_{2}\right\} \cdots\left\{\nu_{\ell-1}, \nu_{\ell}\right\}
$$

where the inner sum is over pairs $(\nu, i)$, where $\nu$ is a matching satisfying (i)-(iii), and $i$ is the index of a double-barred part of $\nu$. Also,

$$
2 m T_{2 m+1, v+1-m}^{0}=\sum_{\mu \in C(\lambda, 2 m+3, v+1-m)} \sum_{(\rho, j)} \epsilon(\lambda, \rho)\left\{\rho_{1}, \rho_{2}\right\} \cdots\left\{\rho_{\ell-1}, \rho_{\ell}\right\},
$$

where the inner sum is over pairs $(\rho, j)$, where $\rho$ is a matching satisfying (i)-(iii), and where $j$ is the index of a single-barred part of $\rho$, which is not in the distinguished pair, nor in the unique pair which contains only one bar. Observe that there is a bijection between the pairs $(\nu, i)$ which appear in (32) and pairs $(\rho, j)$ in (33).

Adding (32) and (33) and using the bijection between the indexing sets and the identity

$$
(\overline{\bar{c}}, d)+2(\bar{c}, \bar{d})+(c, \overline{\bar{d}})=[\bar{c}, d]+[c, \bar{d}]
$$


we may express the left-hand side of (31) as a sum in which every summand has two distinguished pairs. By invoking the identity

$$
\begin{aligned}
{[\bar{a}, \bar{b}]([\bar{c}, d]+[c, \bar{d}])-[\bar{a}, \bar{c}]([\bar{b}, d]+[b, \bar{d}]) } & +[\bar{a}, \bar{b}]([\bar{b}, c]+[b, \bar{c}]) \\
= & -(\overline{\bar{a}})(\overline{\bar{b}})[c, d]+(\overline{\bar{a}})(\overline{\bar{c}})[b, d]-(\overline{\bar{a}})(\overline{\bar{d}})[b, c]
\end{aligned}
$$

(with $a=\lambda_{\ell}$, always), we reduce ourselves to a situation where we may use Lemma 2 and conclude that (31) holds.

It follows from Lemma 4 and the previous discussion that (29) holds for $g=0$. When $g$ is positive, the above argument goes through with the triple $(b, r, s)$ replaced with $(b-2 g, r-2 g, s-2 g)$; note that the binomial coefficients in (29) remain unchanged under these substitutions. This finishes the proof of Theorem 3

\section{Isotropic Grassmannians}

3.1. Schubert subvarieties and incidence loci. The principal object of study is the Lagrangian Grassmannian $L G(n, 2 n)$ which parametrizes $n$-dimensional subspaces of a fixed $2 n$-dimensional complex vector space $V$, isotropic for a fixed nondegenerate skew-symmetric bilinear form on $V$. When $n$ is fixed, we write $L G$ for $L G(n, 2 n)$. We have $\operatorname{dim}_{\mathbb{C}} L G=n(n+1) / 2$. The identities in cohomology that we establish in 3.1 and 3.2 remain valid if we work over an arbitrary base field, using Chow rings in place of cohomology.

Let $F_{\bullet}$ be a fixed complete isotropic flag of subspaces of $V$. The Schubert varieties $\mathfrak{X}_{\lambda} \subset L G$ - defined relative to $F_{\bullet}-$ are indexed by partitions $\lambda \in \mathcal{D}_{n}$. One has $\operatorname{dim}\left(\Sigma \cap F_{k}^{\perp}\right)=\operatorname{dim}\left(\Sigma \cap F_{k}\right)+n-k$ for any Lagrangian subspace $\Sigma$, so there are two ways to write the conditions which define a Schubert variety in $L G$ :

$$
\begin{aligned}
\mathfrak{X}_{\lambda} & =\left\{\Sigma \in L G \mid \operatorname{rk}\left(\Sigma \rightarrow V / F_{n+1-\lambda_{i}}\right) \leqslant n-i, i=1, \ldots, \ell(\lambda)\right\} \\
& =\left\{\Sigma \in L G \mid \operatorname{rk}\left(\Sigma \rightarrow V / F_{n+1-\lambda_{i}}^{\perp} \leqslant n+1-i-\lambda_{i}, i=1, \ldots, \ell(\lambda)\right\} .\right.
\end{aligned}
$$

We call particular attention to the alternative formulation (35), as these are the relevant rank conditions for situations where the morphism $\Sigma \rightarrow V$ is allowed to degenerate.

Set $\sigma_{\lambda}=\left[\mathfrak{X}_{\lambda}\right]$ in $H^{*}(L G, \mathbb{Z})$. The classical Giambelli formula (5) for $L G$ is equivalent to the following identity in $H^{*}(L G, \mathbb{Z})$ :

$$
\sigma_{\lambda}=\sum_{j=1}^{r-1}(-1)^{j-1} \sigma_{\lambda_{j}, \lambda_{r}} \cdot \sigma_{\lambda \backslash\left\{\lambda_{j}, \lambda_{r}\right\}},
$$

for $r=2\lfloor(\ell(\lambda)+1) / 2\rfloor$. For $\mu \in \mathcal{D}_{n}$, let us denote $\mu^{\prime}=\rho_{n} \backslash \mu$, the dual partition. Then, we recall, the Poincaré duality pairing on $L G$ satisfies

$$
\int_{L G} \sigma_{\lambda} \sigma_{\mu}=\delta_{\lambda \mu^{\prime}}
$$

Later on, in place of $\Sigma \rightarrow V$, we will have a morphism of vector bundles $\mathcal{E} \rightarrow$ $\mathcal{O}_{T} \otimes V$ over some base $T$, with $\mathcal{E}$ a rank $n$ vector bundle and with the morphism generically of full rank, but with loci where the rank drops. So we need to study the Grassmannians $I G(k, 2 n)$ of isotropic $k$-dimensional subspaces of $V$, for various $k<n$, notably for $k=n-1$ and $k=n-2$. We have $I G(n, 2 n)=L G(n, 2 n)$.

Observe that any $S p_{2 n}$-translate of the Schubert variety $\mathfrak{X}_{n, n-1, \ldots, k+1}$ in $L G$ is of the form $\{\Sigma \in L G \mid \Sigma \supset A\}$ for a unique $A \in I G(n-k, 2 n)$. Any such translate 
can be identified with $L G(k, 2 k)$, and moreover, for any $\lambda$, meets $\mathfrak{X}_{\lambda}$ in a Schubert subvariety of $L G(k, 2 k)$ :

Proposition 3. Let $A$ be an isotropic subspace of $V$ of dimension $n-k$, and let $Y \subset L G(n, 2 n)$ be the subvariety of Lagrangian subspaces of $V$ which contain $A$. Then $\mathfrak{X}_{\lambda} \cap Y$ is a Schubert variety in $Y \simeq L G(k, 2 k)$ for any $\lambda \in \mathcal{D}_{n}$. Moreover, if $\ell(\lambda)<k$ then the intersection, if nonempty, has positive dimension.

Proof. Define the isotropic flag $\widetilde{F} \bullet$ of subspaces of $A^{\perp} / A$ by $\widetilde{F}_{i}=\left(\left(F_{i}+A\right) \cap A^{\perp}\right) / A$. For any Lagrangian $\Sigma \subset V$ containing $A$, we have $\operatorname{dim}\left(\Sigma \cap F_{i}\right)=\operatorname{dim}\left((\Sigma / A) \cap \widetilde{F}_{i}\right)+$ $\operatorname{dim}\left(A \cap F_{i}\right)$. So $\mathfrak{X}_{\lambda} \cap Y$ is defined by the attitude of $\Sigma / A$ with respect to $\widetilde{F}_{\bullet}$, and hence is a Schubert variety (if nonempty). For the intersection to be a point, we would require at least $k$ rank conditions as in (34), that is, $\ell(\lambda) \geqslant k$.

The space $I G(n-1,2 n)$ is the parameter space of lines on $L G$, and the variety of lines incident to $\mathfrak{X}_{\lambda}$ (for nonempty $\lambda$ ) is the Schubert variety

$$
\mathfrak{X}_{\lambda}^{\prime}=\left\{\Sigma^{\prime} \in I G(n-1,2 n) \mid \operatorname{rk}\left(\Sigma^{\prime} \rightarrow V / F_{n+1-\lambda_{i}}^{\perp}\right) \leqslant n+1-i-\lambda_{i} \text {, all } i\right\} .
$$

Note the rank conditions are identical to those in (35). The codimension of $\mathfrak{X}_{\lambda}^{\prime}$ is $|\lambda|-1$. Analogously, $I G(n-2,2 n)$ parametrizes translates of $\mathfrak{X}_{n \cdots 43}$ (isomorphic to the quadric threefold $L G(2,4))$ on $L G$. Now suppose $\ell(\lambda) \geqslant 2$; then translates of $\mathfrak{X}_{n \cdots 43}$ on $L G$ incident to $\mathfrak{X}_{\lambda}$ form the Schubert variety

$$
\mathfrak{X}_{\lambda}^{\prime \prime}=\left\{\Sigma^{\prime \prime} \in I G(n-2,2 n) \mid \operatorname{rk}\left(\Sigma^{\prime \prime} \rightarrow V / F_{n+1-\lambda_{i}}^{\perp}\right) \leqslant n+1-i-\lambda_{i}, \text { all } i\right\},
$$

which has codimension $|\lambda|-3$. We remark that the subvarieties we have described are only some of the Schubert varieties in $I G(n-1,2 n)$ and $I G(n-2,2 n)$.

The modular interpretation of the loci $\mathfrak{X}_{\lambda}^{\prime}$ and $\mathfrak{X}_{\lambda}^{\prime \prime}$ is explained by

Lemma 5. Let $A$ be an isotropic subspace of $V$ of dimension $n-k$, and let $F_{n}$ be a fixed Lagrangian subspace of $V$. Then there exists a unique Lagrangian subspace $\Sigma$ which contains $A$ and satisfies $\operatorname{dim}\left(\Sigma \cap F_{n}\right)=\operatorname{dim}\left(A \cap F_{n}\right)+k$. In fact, we have $\Sigma=\operatorname{Span}\left(A, A^{\perp} \cap F_{n}\right)$.

Proof. Set $W=A \cap F_{n}$; replacing $V$ by $W^{\perp} / W$ and $A$ and $F_{n}$ by their respective images in $W^{\perp} / W$, we are reduced to the case $A \cap F_{n}=0$. Now for dimension reasons, we have $\operatorname{dim}\left(A^{\perp} \cap F_{n}\right) \geqslant k$, so isotropicity of $\operatorname{Span}\left(A, A^{\perp} \cap F_{n}\right)$ forces $\operatorname{dim}\left(A^{\perp} \cap F_{n}\right)=k$, and the assertion is clear.

3.2. Pfaffian identities on isotropic Grassmannians. Let $F=F_{S p}(V)$ denote the variety of complete isotropic flags in $V$. There are natural projection maps from $F$ to the Grassmannians $I G(n-1,2 n)$ and $I G(n-2,2 n)$, inducing injective pullback morphisms on cohomology. Referring to KT1, §2.4], one sees that the Schubert class $\left[\mathfrak{X}_{\lambda}^{\prime}\right]$ (resp. $\left.\left[\mathfrak{X}_{\lambda}^{\prime \prime}\right]\right)$ in $H^{*}(I G(n-1,2 n))$ (resp. $H^{*}(I G(n-2,2 n))$ pulls back to the class $\mathfrak{C}_{\lambda}^{\prime}(X)$ (resp. $\left.\mathfrak{C}_{\lambda}^{\prime \prime}(X)\right)$ in $H^{*}(F)$, for each $\lambda \in \mathcal{D}_{n}$. Here $X=\left(x_{1}, \ldots, x_{n}\right)$ is the vector of Chern roots of the dual to the tautological rank $n$ vector bundle over $F$, ordered as in [KT1 Sect. 2]. Theorems 2] and [ now give

Corollary 1. a) For every $\lambda \in \mathcal{D}_{n}$ of length $\ell \geqslant 3$ and $r=2\lfloor(\ell+1) / 2\rfloor$ we have

$$
\sum_{j=1}^{r-1}(-1)^{j-1}\left[\mathfrak{X}_{\lambda_{j}, \lambda_{r}}^{\prime}\right]\left[\mathfrak{X}_{\lambda \backslash\left\{\lambda_{j}, \lambda_{r}\right\}}^{\prime}\right]=0
$$

in $H^{*}(I G(n-1,2 n), \mathbb{Z})$. 
b) For every $\lambda \in \mathcal{D}_{n}$ of even length $\ell \geqslant 4$ we have

$$
\sum_{j=1}^{\ell-1}(-1)^{j-1}\left[\mathfrak{X}_{\lambda_{j}, \lambda_{\ell}}^{\prime \prime}\right]\left[\mathfrak{X}_{\lambda \backslash\left\{\lambda_{j}, \lambda_{\ell}\right\}}^{\prime \prime}\right]=0
$$

in $H^{*}(I G(n-2,2 n), \mathbb{Z})$.

3.3. Counting lines on $L G(n, 2 n)$. In this subsection, we exploit a correspondence between lines on $L G(n, 2 n)$ and points on $L G(n+1,2 n+2)$ to derive the following formula for three-point degree-1 Gromov-Witten invariants on $L G=$ $L G(n, 2 n)$ :

Proposition 4. For $\lambda, \mu, \nu \in \mathcal{D}_{n}$ we have

$$
\left\langle\sigma_{\lambda}, \sigma_{\mu}, \sigma_{\nu}\right\rangle_{1}=\frac{1}{2} \int_{L G(n+1,2 n+2)}\left[\mathfrak{X}_{\lambda}^{+}\right] \cdot\left[\mathfrak{X}_{\mu}^{+}\right] \cdot\left[\mathfrak{X}_{\nu}^{+}\right],
$$

where $\mathfrak{X}_{\lambda}^{+}, \mathfrak{X}_{\mu}^{+}, \mathfrak{X}_{\nu}^{+}$denote Schubert varieties in $L G(n+1,2 n+2)$.

Proof. Let $V$ be as in 3.1 let $H$ be a 2-dimensional symplectic vector space and let $V^{+}$be the orthogonal direct sum of $V$ and $H$. Consider the correspondence between $L G(n+1,2 n+2)$ and $I G(n-1,2 n)$ consisting of pairs $\left(\Sigma^{+}, \Sigma^{\prime}\right)$ with $\Sigma^{+}$ a Lagrangian subspace of $V^{+}$and $\Sigma^{\prime}$ an isotropic $(n-1)$-dimensional subspace of $V$, given by the condition $\Sigma^{\prime} \subset \Sigma^{+}$. This is the correspondence induced by the rational map which sends $\left[\Sigma^{+}\right]$to $\left[\Sigma^{+} \cap V\right]$, defined for Lagrangian $\Sigma^{+} \subset V^{+}$with $\operatorname{dim}\left(\Sigma^{+} \cap V\right)=n-1$. Choose a nonzero $h \in H$ and consider the isotropic flags $F_{\bullet}$ in $V$ and $F_{\bullet}^{+}$in $V^{+}$, with $F_{i}^{+}=F_{i-1} \oplus\langle h\rangle$. Then, for any $\lambda \in \mathcal{D}_{n}$, we have that to any $\Sigma^{+}$with $\Sigma^{+} \in \mathfrak{X}_{\lambda}^{+} \subset L G(n+1,2 n+2)$, there corresponds $\Sigma^{\prime}$ with $\Sigma^{\prime} \in \mathfrak{X}_{\lambda}^{\prime}$.

The degree of $q$ in $Q H^{*}(L G(n, 2 n))$ equals

$$
\int_{L G} c_{1}\left(T_{L G}\right) \cdot \sigma_{1^{\prime}}=n+1
$$

so for degree reasons, the Gromov-Witten invariant $\left\langle\sigma_{\lambda}, \sigma_{\mu}, \sigma_{\nu}\right\rangle_{1}$ is potentially nonzero (and hence counts lines on $L G$ ) when

$$
|\lambda|+|\mu|+|\nu|=(n+1)(n / 2+1) .
$$

Now choose general translates of isotropic flags of subspaces of $V$ such that $\mathfrak{X}_{\lambda}^{\prime}, \mathfrak{X}_{\mu}^{\prime}$, and $\mathfrak{X}_{\nu}^{\prime}$ meet transversely, and such that every point in the intersection corresponds to a line in $L G(n, 2 n)$ which

(i) is incident to $\mathfrak{X}_{\lambda}, \mathfrak{X}_{\mu}$, and $\mathfrak{X}_{\nu}$ at three distinct points;

(ii) has each incidence point in the corresponding Schubert cell inside the Schubert variety (i.e., all dimension estimates are sharp);

(iii) (for $\ell=\ell(\lambda)<n$ ) the line is not incident to $\mathfrak{X}_{\rho_{\ell+1}}$, and similarly for $\mu$ and $\nu$ (using the respective defining flags).

We remark that (ii) and (iii) can be combined into the single statement, that the points in $\mathfrak{X}_{\lambda}^{\prime} \cap \mathfrak{X}_{\mu}^{\prime} \cap \mathfrak{X}_{\nu}^{\prime}$ all lie in the intersections of the corresponding Schubert cells in $I G(n-1,2 n)$.

For each of $\lambda, \mu, \nu$, we extend the corresponding flag of isotropic spaces in $V$ to subspaces of $V^{+}$by adjoining, in each case, a generally chosen element of $H$. Now it is evident that every point in $\mathfrak{X}_{\lambda}^{+} \cap \mathfrak{X}_{\mu}^{+} \cap \mathfrak{X}_{\nu}^{+}$(intersection on $L G(n+1,2 n+2)$ ) corresponds to a point in $\mathfrak{X}_{\lambda}^{\prime} \cap \mathfrak{X}_{\mu}^{\prime} \cap \mathfrak{X}_{\nu}^{\prime}$. Analysis using Lemma 5 shows that to every point in $\mathfrak{X}_{\lambda}^{\prime} \cap \mathfrak{X}_{\mu}^{\prime} \cap \mathfrak{X}_{\nu}^{\prime}$ there correspond exactly two points in $\mathfrak{X}_{\lambda}^{+} \cap \mathfrak{X}_{\mu}^{+} \cap \mathfrak{X}_{\nu}^{+}$, 
each a point of transverse intersection. This uses the fact that on $L G(2,4)$ (case $n=1$ ), three general $S L_{2} \times S L_{2}$ translates of $\mathfrak{X}_{1}^{+}$meet transversely at two points, both away from the locus of $\Sigma^{+}$meeting $V$ nontrivially.

Corollary 2. If $i$ and $j$ are such that $i+j \geqslant n+1$, then

$$
\sigma_{i} \sigma_{j}=2 \sum_{k=1}^{n-i} \sigma_{i+k, j-k}+\sigma_{i+j-n-1} q
$$

in $Q H^{*}(L G(n, 2 n))$.

From the Corollary we immediately deduce that the quantum relation (3) and the two-condition quantum Giambelli formula (44) are valid in $Q H^{*}(L G)$. By [ST], now, we obtain a presentation of $Q H^{*}(L G)$ as a quotient of the polynomial ring $\mathbb{Z}\left[\sigma_{1}, \ldots, \sigma_{n}, q\right]$ modulo the relations (3) (see also [FP] Sect. 10]). The proof of the more difficult relation (5) occupies Sections 4 and 5

\section{Lagrangian QuOT SCHEMES}

4.1. Overview. Here is a summary of our analysis in the next two sections. The goal is to establish an identity in the Chow group of the Lagrangian Quot schemes, from which identity (5) in $Q H^{*}(L G(n, 2 n))$ follows. We make use of type $C$ degeneracy loci for isotropic morphisms of vector bundles KT1 to define classes $\left[W_{\lambda}(p)\right]_{k}$ $\left(p \in \mathbb{P}^{1}\right)$ of the appropriate dimension $k:=(n+1)(n / 2+d)-|\lambda|$ in the Chow group of the Quot scheme $L Q_{d}$, which compactifies the space of degree- $d$ maps $\mathbb{P}^{1} \rightarrow L G$. Choose a point $p^{\prime}$ on $\mathbb{P}^{1}$ distinct from $p$, and use $\widetilde{W}$ to denote a degeneracy locus defined with respect to a general translate of the fixed isotropic flag of vector spaces. We produce a Pfaffian formula analogous to (36):

$$
\left[W_{\lambda}(p)\right]_{k}=\sum_{j=1}^{r-1}(-1)^{j-1}\left[W_{\lambda_{j}, \lambda_{r}}(p) \cap \widetilde{W}_{\lambda \backslash\left\{\lambda_{j}, \lambda_{r}\right\}}\left(p^{\prime}\right)\right]_{k},
$$

for any $\lambda \in \mathcal{D}_{n}$ with $\ell(\lambda) \geqslant 3$ and $r=2\lfloor(\ell(\lambda)+1) / 2\rfloor$.

In fact, we need the cycles in (41) to remain rationally equivalent under further intersection with some (general translate of) $W_{\mu}\left(p^{\prime \prime}\right)$ (with varying $\mu$, where $p^{\prime \prime}$ is a third distinct point on $\left.\mathbb{P}^{1}\right)$. The way around the difficulty of a lack of an intersection product on the (possibly singular) $L Q_{d}$ is to work instead on a modification $L Q_{d}\left(p^{\prime \prime}\right)$, on which the evaluation-at- $p^{\prime \prime}$ map is globally defined, and employ a refined intersection operation from $L G$. Then, the rational equivalence implies that the (zero!) number of degree- $d$ maps $\mathbb{P}^{1} \rightarrow L G$ which send $p$ to $\mathfrak{X}_{\lambda}$ and $p^{\prime \prime}$ to a general translate of $\mathfrak{X}_{\mu}$ - with no constraint on any third point - is equal to the alternating sum of 3 -point degree- $d$ structure constants $\left\langle\sigma_{\lambda_{j}, \lambda_{r}}, \sigma_{\lambda_{\backslash}}\left\{\lambda_{j}, \lambda_{r}\right\}, \sigma_{\mu}\right\rangle_{d}$, and this is exactly what we require to establish quantum Giambelli (Theorem 5).

The required rational equivalence (which is similar to (41), but involves cycles on $L Q_{d}\left(p^{\prime \prime}\right)$ ) is a sum of rational equivalences coming from three sources: (i) Pfaffian formulas on $L G$ (36); (ii) supplementary rational equivalences on the isotropic Grassmannians of $(n-1)$-dimensional and $(n-2)$-dimensional isotropic subspaces of the ambient vector space (39), (40); (iii) rational equivalences $\{p\} \sim\left\{p^{\prime}\right\}$ on $\mathbb{P}^{1}$. The most interesting of these are (ii). Indeed, at some stage in the analysis, we consider intersections $W_{\lambda_{j}, \lambda_{r}}(p) \cap \widetilde{W}_{\lambda \backslash\left\{\lambda_{j}, \lambda_{r}\right\}}(p)$, where the same point $p$ appears twice. Such an intersection has several components of the correct dimension. Of 
course, degree- $d$ morphisms $\mathbb{P}^{1} \rightarrow L G$ sending $p$ into $\mathfrak{X}_{\lambda_{j}, \lambda_{r}} \cap \widetilde{\mathfrak{X}}_{\left.\lambda_{\backslash} \backslash \lambda_{j}, \lambda_{r}\right\}}$ make up one such component. But also there are components of the same dimension, entirely contained in the boundary of the Quot scheme. The formulas in Corollary 11 give us precisely the cancellation of these extra contributions.

4.2. Definition of $L Q_{d}$. Fix a complex vector space $V$ and set $N=\operatorname{dim} V$. Let $m$ and $n$ be positive integers, with $m+n=N$. We recall (from [G1]) that the Quot scheme $Q_{d}(d \geqslant 0)$ parametrizing quotient sheaves of $\mathcal{O}_{\mathbb{P}^{1}} \otimes V$ with Hilbert polynomial $n t+n+d$ is a smooth projective variety which compactifies the space of parametrized degree- $d$ maps from $\mathbb{P}^{1}$ to the Grassmannian of $m$-dimensional subspaces of $V$. On $\mathbb{P}^{1} \times Q_{d}$ there is a universal exact sequence of sheaves

$$
0 \longrightarrow \mathcal{E} \longrightarrow \mathcal{O} \otimes V \longrightarrow \mathcal{Q} \longrightarrow 0
$$

with $\mathcal{E}$ locally free of rank $m$.

We fix $m=n$ and $N=2 n$ from now on, and let the $2 n$-dimensional vector space $V$ be endowed with a nondegenerate skew-symmetric bilinear form.

Definition 1. Let $d$ be a nonnegative integer. The Lagrangian Quot scheme $L Q_{d}$ is the closed subscheme of $Q_{d}$ which is defined by the vanishing of the composite

$$
\mathcal{E} \longrightarrow \mathcal{O}_{\mathbb{P}^{1}} \otimes V \stackrel{\alpha}{\longrightarrow} \mathcal{O}_{\mathbb{P}^{1}} \otimes V^{*} \longrightarrow \mathcal{E}^{*}
$$

where $\alpha$ is the isomorphism defined by the symplectic form on $V$.

For $n=2$, the scheme $L Q_{1}$ was introduced and studied in KT1; it is nonsingular. For general $n$ and $d$, however, $L Q_{d}$ has singularities. The open subscheme $L M_{d} \subset L Q_{d}$, defined as the locus where $\mathcal{E} \rightarrow \mathcal{O}_{\mathbb{P}^{1}} \otimes V$ has everywhere full rank, is smooth and irreducible, and coincides with the moduli space $M_{0,3}(L G, d)$ of 3pointed (that is, parametrized) maps $\mathbb{P}^{1} \rightarrow L G$ of degree $d$ (the irreducibility of all $M_{0, i}(L G, d)$ is known [KP] Th] $)$.

4.3. Degeneracy loci. Degeneracy loci for isotropic morphisms of vector bundles in type $C$ (as well as types $B$ and $D$ ) were introduced in [KT1.

Definition 2. The degeneracy loci $W_{\lambda}$ and $W_{\lambda}(p)\left(\lambda \in \mathcal{D}_{n}\right.$ and $\left.p \in \mathbb{P}^{1}\right)$ are the following subschemes of $\mathbb{P}^{1} \times L Q_{d}$ :

$$
\begin{gathered}
W_{\lambda}=\left\{x \in \mathbb{P}^{1} \times L Q_{d} \mid \operatorname{rk}\left(\mathcal{E} \rightarrow \mathcal{O} \otimes V / F_{n+1-\lambda_{i}}^{\perp}\right)_{x} \leqslant n+1-i-\lambda_{i},\right. \\
i=1, \ldots, \ell(\lambda)\}, \\
W_{\lambda}(p)=W_{\lambda} \cap\left(\{p\} \times L Q_{d}\right)
\end{gathered}
$$

Define also

$$
h(n, d)=(n+1)(n / 2+d)
$$

the dimension of the Lagrangian Quot scheme $L Q_{d}$. The fact that three-term Gromov-Witten invariants on $L G$ arise as numbers of points in intersections of degeneracy loci on $L Q_{d}$ is a consequence of the

Moving Lemma. Let $k$ be a positive integer, and let $p_{1}, \ldots, p_{k}$ be distinct points on $\mathbb{P}^{1}$. Let $\lambda^{1}, \ldots, \lambda^{k}$ be partitions in $\mathcal{D}_{n}$, and let us take the degeneracy loci $W_{\lambda^{1}}\left(p_{1}\right), \ldots, W_{\lambda^{k}}\left(p_{k}\right)$ to be defined by isotropic flags of vector spaces in general position. Let us define

$$
Z=W_{\lambda^{1}}\left(p_{1}\right) \cap \cdots \cap W_{\lambda^{k}}\left(p_{k}\right)
$$


Then $Z$ has dimension at most $h(n, d)-\sum_{i=1}^{k}\left|\lambda^{i}\right|$. Moreover, $Z \cap L M_{d}$ is either empty or generically reduced and of pure dimension $h(n, d)-\sum_{i}\left|\lambda^{i}\right|$; also, $Z \cap$ $\left(L Q_{d} \backslash L M_{d}\right)$ has dimension at most $h(n, d)-\sum_{i=1}^{k}\left|\lambda^{i}\right|-1$.

We prove the Moving Lemma in 4.4 after stating a theorem which describes the structure of the boundary of $L Q_{d}$.

Corollary 3. Let $p, p^{\prime}, p^{\prime \prime} \in \mathbb{P}^{1}$ be distinct points. Suppose $\lambda, \mu, \nu \in \mathcal{D}_{n}$ satisfy $|\lambda|+|\mu|+|\nu|=h(n, d)$. With degeneracy loci defined with respect to isotropic flags in general position, the intersection $W_{\lambda}(p) \cap W_{\mu}\left(p^{\prime}\right) \cap W_{\nu}\left(p^{\prime \prime}\right)$ consists of finitely many reduced points, all contained in $L M_{d}$, and the corresponding Gromov-Witten invariant on $L G$ equals the cardinality of this set of points:

$$
\left\langle\sigma_{\lambda}, \sigma_{\mu}, \sigma_{\nu}\right\rangle_{d}=\#\left(W_{\lambda}(p) \cap W_{\mu}\left(p^{\prime}\right) \cap W_{\nu}\left(p^{\prime \prime}\right)\right) .
$$

Corollary 4. If $p$ and $p^{\prime}$ are distinct points of $\mathbb{P}^{1}$ and if $|\lambda|+|\mu|=h(n, d)$, then $W_{\lambda}(p) \cap \widetilde{W}_{\mu}\left(p^{\prime}\right)=\emptyset$ for a general translate $\widetilde{W}_{\mu}\left(p^{\prime}\right)$ of $W_{\mu}\left(p^{\prime}\right)$.

As in Bertram's paper $\mathrm{Be}$, we cover $L Q_{d}$ by images of Grassmann bundles over smaller Quot schemes.

Definition 3. We let $\pi_{c}: G_{c} \rightarrow \mathbb{P}^{1} \times L Q_{d-c}$ denote the Grassmann bundle of $c$ dimensional quotients of the universal bundle $\mathcal{E}$ on $\mathbb{P}^{1} \times L Q_{d-c}$. The morphism $\beta_{c}: G_{c} \rightarrow L Q_{d}$ is given as follows. On $G_{c}$ there is the universal quotient bundle $\mathcal{F}_{c}$, of rank $c$. If $i_{c}$ denotes the morphism $G_{c} \rightarrow \mathbb{P}^{1} \times G_{c}$ given by $\left(\mathrm{pr}_{1} \circ \pi_{c}\right.$, id), then there is a natural morphism of sheaves $\left(\mathrm{id} \times\left(\mathrm{pr}_{2} \circ \pi_{c}\right)\right)^{*} \mathcal{E} \rightarrow i_{c *} \pi_{c}^{*} \mathcal{E}$ on $\mathbb{P}^{1} \times G_{c}$. We define $\mathcal{E}_{c}$ to be the kernel of this morphism composed with $i_{c *}$ of the morphism to the universal quotient bundle on $G_{c}$ :

$$
\mathcal{E}_{c}=\operatorname{ker}\left(\left(\mathrm{id} \times\left(\mathrm{pr}_{2} \circ \pi_{c}\right)\right)^{*} \mathcal{E} \rightarrow i_{c *} \mathcal{F}_{c}\right) .
$$

Now $\mathcal{E}_{c}$ is a subsheaf of $\mathcal{O} \otimes V$ on $\mathbb{P}^{1} \times G_{c}$, with cokernel flat over $G_{c}$, and hence there is an induced morphism $\beta_{c}: G_{c} \rightarrow L Q_{d}$.

It is natural to consider degeneracy loci with respect to the bundles $\mathcal{E}_{c}$.

Definition 4. The degeneracy loci $\widehat{W}_{c, \lambda}$ and $\widehat{W}_{c, \lambda}(p)\left(\lambda \in \mathcal{D}_{n}\right.$ and $\left.p \in \mathbb{P}^{1}\right)$ are the following subschemes of $G_{c}$ :

$$
\begin{aligned}
\widehat{W}_{c, \lambda} & =\left\{x \in G_{c} \mid \operatorname{rk}\left(\mathcal{E}_{c} \rightarrow \mathcal{O} \otimes V / F_{n+1-\lambda_{i}}^{\perp}\right)_{x} \leqslant n+1-i-\lambda_{i},\right. \\
i=1, \ldots, \ell(\lambda)\}, & \\
\widehat{W}_{c, \lambda}(p) & =\widehat{W}_{c, \lambda}(p) \cap \pi_{c}^{-1}\left(\{p\} \times L Q_{d-c}\right)
\end{aligned}
$$

4.4. Boundary structure of $L Q_{d}$. The boundary of $L Q_{d}$ is made up of points at which $\mathcal{Q}$ fails to be locally free, or equivalently, where $\mathcal{E} \rightarrow \mathcal{O} \otimes V$ drops rank at one or more points of $\mathbb{P}^{1}$. The following theorem is taken more-or-less verbatim from [Be].

Theorem 4. The maps $\beta_{c}: G_{c} \rightarrow L Q_{d}$ satisfy

(i) Given $x \in L Q_{d}$, if $\mathcal{Q}_{x}$ has rank at least $n+c$ at $p \in \mathbb{P}^{1}$, then $x$ lies in the image of $\beta_{c}$.

(ii) The restriction of $\beta_{c}$ to $\pi_{c}^{-1}\left(\mathbb{P}^{1} \times L M_{d-c}\right)$ is a locally closed immersion.

(iii) We have

$$
\beta_{c}^{-1}\left(W_{\lambda}(p)\right)=\pi_{c}^{-1}\left(\mathbb{P}^{1} \times W_{\lambda}(p)\right) \cup \widehat{W}_{c, \lambda}(p)
$$

where on the right, $W_{\lambda}(p)$ denotes the degeneracy locus in $L Q_{d-c}$. 
Proof. The argument is exactly as in $[\mathrm{Be}]$.

Proof of the Moving Lemma. The assertions on $L M_{d}$ are clear by transversality of general translates (cf. [H] III.10.8]). So, we restrict attention to the boundary; it is enough to show that

$$
\pi_{c}^{-1}\left(\mathbb{P}^{1} \times \bigcap_{i=1}^{k} W_{\lambda^{i}}\left(p_{i}\right)\right) \text { and } \pi_{c}^{-1}\left(\mathbb{P}^{1} \times \bigcap_{\substack{i=1 \\ i \neq j}}^{k} W_{\lambda^{i}}\left(p_{i}\right)\right) \cap \widehat{W}_{c, \lambda^{j}}\left(p_{j}\right) \quad(j=1, \ldots, k)
$$

have dimension at most $h(n, d)-\sum_{i}\left|\lambda^{i}\right|-1$. The first of these is taken care of by induction on the degree $d$. For the second: there are loci

$$
U_{c}(p) \subset \pi_{c}^{-1}\left(\{p\} \times L Q_{d-c}\right)
$$

defined by the condition $\operatorname{rk}\left(\mathcal{E}_{c} \rightarrow \mathcal{O} \otimes V\right)=n-c$, and evaluation maps

$$
f_{c}^{p}: U_{c}(p) \rightarrow I G(n-c, 2 n) .
$$

Given $x \in L Q_{d}$, if $\mathcal{Q}_{x}$ has rank $n+c$ then $x \in \beta_{c}\left(U_{c}(p)\right)$, so it suffices to prove dimension estimates for

$$
\pi_{c}^{-1}\left(\mathbb{P}^{1} \times \bigcap_{\substack{i=1 \\ i \neq j}}^{k} W_{\lambda^{i}}\left(p_{i}\right)\right) \cap \widehat{W}_{c, \lambda j}\left(p_{j}\right) \cap U_{c}\left(p_{j}\right) .
$$

But now $\widehat{W}_{c, \lambda^{j}}\left(p_{j}\right) \cap U_{c}\left(p_{j}\right)=\left(f_{c}^{p}\right)^{-1}\left(\mathfrak{X}^{\prime}\right)$ for a Schubert variety $\mathfrak{X}^{\prime}$ in $I G(n-c, 2 n)$ of codimension at least $\left|\lambda^{j}\right|-c(c+1) / 2$. Now, by transversality of a general translate, the intersection (42) has dimension at most $h(n, d)-1-\sum_{i}\left|\lambda^{i}\right|$.

\section{Intersections on $L Q_{d}$ And the QuANtum Giambelli formula}

As outlined in 4.1 we need three kinds of rational equivalences on $L Q_{d}$. Two of the rational equivalences we require follow from a simple intersection-theoretic lemma, while in the remainder of this section we develop the third kind. The Chow group (algebraic cycles modulo rational equivalence) of a scheme $\mathfrak{X}$ is denoted $A_{*} \mathfrak{X}$. We employ the following notation.

Definition 5. Let $p$ denote a point of $\mathbb{P}^{1}$ :

(i) $\mathrm{ev}^{p}: L M_{d} \rightarrow L G$ is the evaluation at $p$ morphism;

(ii) $\tau(p): L Q_{d}(p) \rightarrow L Q_{d}$ is the projection from the relative Lagrangian Grassmannian $L Q_{d}(p):=L G_{n}\left(\left.\mathcal{Q}\right|_{\{p\} \times L Q_{d}}\right)$, that is, the closed subscheme of the Grassmannian $\operatorname{Grass}_{n}$ of rank- $n$ quotients G2 of the indicated coherent sheaf, defined by the isotropicity condition on the kernel of the composite morphism from $\mathcal{O}_{\operatorname{Grass}_{n}} \otimes V$ to the universal quotient bundle of the relative Grassmannian;

(iii) $\operatorname{ev}(p): L Q_{d}(p) \rightarrow L G$ is the evaluation morphism on the relative Lagrangian Grassmannian;

(iv) $\mathrm{ev}_{c}^{p}: \pi_{c}^{-1}\left(\{p\} \times L M_{d-c}\right) \rightarrow I G(n-c, 2 n)$ is evaluation at $p$.

Lemma 6. Let $T$ be a projective variety which is a homogenous space for an algebraic group $G$. Let $\mathfrak{X}$ be a scheme, equipped with an action of the group $G$. Let $U$ be a $G$-invariant integral open subscheme of $\mathfrak{X}$, and let $f: U \rightarrow T$ be a $G$-equivariant morphism. Then the map on algebraic cycles

$$
[V] \mapsto\left[f^{-1}(V)^{-}\right]
$$


(this sends the cycle of an integral closed subscheme $V$ of $T$ to the cycle associated with the closure in $\mathfrak{X}$ of the pre-image in $U$ ) respects rational equivalence, and hence induces a map on Chow groups $A_{*} T \rightarrow A_{*} \mathfrak{X}$.

Proof. Denote by $Z$ the closure in $\mathfrak{X} \times T$ of the graph of $f$. Since $T$ is projective, the map $Z \rightarrow \mathfrak{X}$ is proper. The action of $G$ on $U$ extends to an action on $Z$. The map $Z \rightarrow T$ is $G$-equivariant, and hence is flat; the restriction to $Z \backslash U$ is flat as well, of smaller relative dimension. It follows that the map on cycles (43) is equal to the composite of flat pullback to $Z$, followed by proper pushforward to $\mathfrak{X}$. Since these operations respect rational equivalence, so does the composite.

Let us denote by $\widetilde{\mathfrak{X}}_{\mu}, \widetilde{\mathfrak{X}}_{\mu}^{\prime}$ and $\widetilde{\mathfrak{X}}_{\mu}^{\prime \prime}$ the translates of $\mathfrak{X}_{\mu}, \mathfrak{X}_{\mu}^{\prime}$ and $\mathfrak{X}_{\mu}^{\prime \prime}$ by a general element of the algebraic group $S p_{2 n}$.

Corollary 5. The following cycles are rationally equivalent to zero on $L Q_{d}$, and also on $L Q_{d}\left(p^{\prime}\right)$, for any $\lambda \in \mathcal{D}_{n}$, with $\ell=\ell(\lambda)$ and $r=2\lfloor(\ell+1) / 2\rfloor$, and distinct points $p, p^{\prime} \in \mathbb{P}^{1}$.

(i) $(\ell \geqslant 3)\left[\left(\mathrm{ev}^{p}\right)^{-1}\left(\mathfrak{X}_{\lambda}\right)^{-}\right]-\sum_{j=1}^{r-1}(-1)^{j-1}\left[\left(\mathrm{ev}^{p}\right)^{-1}\left(\mathfrak{X}_{\lambda_{j}, \lambda_{r}} \cap \widetilde{\mathfrak{X}}_{\lambda \backslash\left\{\lambda_{j}, \lambda_{r}\right\}}\right)^{-}\right]$.

(ii) $(\ell \geqslant 3) \sum_{j=1}^{r-1}(-1)^{j-1}\left[\beta_{1}\left(\left(\operatorname{ev}_{1}^{p}\right)^{-1}\left(\mathfrak{X}_{\lambda_{j}, \lambda_{r}}^{\prime} \cap \widetilde{\mathfrak{X}}_{\lambda \backslash\left\{\lambda_{j}, \lambda_{r}\right\}}^{\prime}\right)\right)^{-}\right]$.

(iii) $(r=\ell \geqslant 4) \sum_{j=1}^{r-1}(-1)^{j-1}\left[\beta_{2}\left(\left(\operatorname{ev}_{2}^{p}\right)^{-1}\left(\mathfrak{X}_{\lambda_{j}, \lambda_{r}}^{\prime \prime} \cap \widetilde{\mathfrak{X}}_{\lambda \backslash\left\{\lambda_{j}, \lambda_{r}\right\}}^{\prime \prime}\right)\right)^{-}\right]$.

Proof. We get (i) from the classical Giambelli formula by invoking Lemma [6 with $\mathfrak{X}=L Q_{d}$ or $\mathfrak{X}=L Q_{d}\left(p^{\prime}\right)$, and $U=L M_{d}$. The rational equivalences of Corollary 1 give us (ii) and (iii): we invoke Lemma $\left[\right.$ with $\mathfrak{X}=\pi_{c}^{-1}\left(\{p\} \times L Q_{d-c}\right)$ or $\mathfrak{X}=$ $\pi_{c}^{-1}\left(\{p\} \times L Q_{d-c}\right) \times_{L Q_{d}} L Q_{d}\left(p^{\prime}\right)$, and $U=\pi_{c}^{-1}\left(\{p\} \times L M_{d-c}\right)$, where $c=1$ for (ii) and $c=2$ for (iii).

Recall that given a closed subscheme $Z$ of a scheme $\mathfrak{X}$, one defines $[Z] \in A_{*} \mathfrak{X}$ to be the class in the Chow group of the formal sum $\sum m_{V}[V]$ where $V$ runs over integral closed subschemes of $\mathfrak{X}$ which are irreducible components of $Z$, and where $m_{V}$ is the geometric multiplicity of $V$ in $Z$. In the following statement, we write $[Z]_{k}$ to denote the $k$-dimensional component of $[Z]$, that is, the class of the same sum with $V$ restricted to the irreducible components of $\mathfrak{X}$ which have dimension $k$.

Proposition 5. (a) Suppose $\lambda$ and $\mu$ are in $\mathcal{D}_{n}$, and let $p, p^{\prime}, p^{\prime \prime}$ be distinct points in $\mathbb{P}^{1}$. Assume $\ell(\lambda)$ equals 1 or 2 and $\ell(\mu) \geqslant 2$. Let $k=h(n, d)-|\lambda|-|\mu|$. Then

$$
\left[W_{\lambda}(p) \cap \widetilde{W}_{\mu}\left(p^{\prime}\right)\right]_{k}=\left[W_{\lambda}(p) \cap \widetilde{W}_{\mu}(p)\right]_{k} \quad \text { in } A_{*} L Q_{d},
$$

$$
\left[\tau\left(p^{\prime \prime}\right)^{-1}\left(W_{\lambda}(p) \cap \widetilde{W}_{\mu}\left(p^{\prime}\right)\right)\right]_{k}=\left[\tau\left(p^{\prime \prime}\right)^{-1}\left(W_{\lambda}(p) \cap \widetilde{W}_{\mu}(p)\right)\right]_{k} \quad \text { in } A_{*} L Q_{d}\left(p^{\prime \prime}\right),
$$

where $\widetilde{W}_{\mu}(p)$ denotes degeneracy locus with respect to a general translate of the isotropic flag of subspaces.

(b) In $A_{*} L Q_{d}$, we have

$$
\begin{aligned}
{\left[W_{\lambda}(p) \cap \widetilde{W}_{\mu}(p)\right]_{k}=} & {\left[\left(\mathrm{ev}^{p}\right)^{-1}\left(\mathfrak{X}_{\lambda} \cap \widetilde{\mathfrak{X}}_{\mu}\right)^{-}\right] } \\
& +\left[\beta_{1}\left(\left(\operatorname{ev}_{1}^{p}\right)^{-1}\left(\mathfrak{X}_{\lambda}^{\prime} \cap \widetilde{\mathfrak{X}}_{\mu}^{\prime}\right)\right)^{-}\right] \\
& +\delta_{\ell, 2}\left[\beta_{2}\left(\left(\operatorname{ev}_{2}^{p}\right)^{-1}\left(\mathfrak{X}_{\lambda}^{\prime \prime} \cap \widetilde{\mathfrak{X}}_{\mu}^{\prime \prime}\right)\right)^{-}\right]
\end{aligned}
$$

(with $\delta_{\ell, 2}$ the Kronecker delta), and in $A_{*} L Q_{d}\left(p^{\prime \prime}\right)$, the cycle class $\left[\tau\left(p^{\prime \prime}\right)^{-1}\left(W_{\lambda}(p) \cap\right.\right.$ $\left.\left.\widetilde{W}_{\mu}(p)\right)\right]_{k}$ is equal to the right-hand side of (46). 
Proof. To prove (44), we consider the subscheme $Y:=\left(\mathbb{P}^{1} \times W_{\lambda}(p)\right) \cap \widetilde{W}_{\mu}$ of $\mathbb{P}^{1} \times L Q_{d}$. By generic flatness and equivariance for automorphisms of $\mathbb{P}^{1}$ fixing the point $p$, the morphism $Y \rightarrow \mathbb{P}^{1}$ is flat over $\mathbb{P}^{1} \backslash\{p\}$. In fact, we claim, the morphism is flat on the complement of some closed subscheme of dimension $<k$. Restricting our attention to the $(k+1)$-dimensional irreducible components of $Y$, now, the rational equivalence $\{p\} \sim\left\{p^{\prime}\right\}$ on $\mathbb{P}^{1}$ pulls back to (44) on $L Q_{d}$.

We justify this claim and at the same time establish part (b). First, for any $c \geqslant 1$, the intersection of two general-position Schubert varieties in $I G(n-c, 2 n)$, defined by the rank conditions in (35) corresponding to the partitions $\lambda$ and $\mu$, has codimension at least $|\lambda|+|\mu|-c(c+1)$, with equality only if $\ell(\lambda) \geqslant c$ by Proposition 3 It now follows, by a dimension count similar to the one in the proof of the Moving Lemma, that the irreducible components of $W_{\lambda}(p) \cap \widetilde{W}_{\mu}(p)$ of dimension $k$ are precisely the ones indicated on the right-hand side of (46). A simple argument using Kontsevich's spaces of stable maps (see, e.g., [FP]) shows that each of these components lies in the closure of $\left(\left(\mathbb{P}^{1} \backslash\{p\}\right) \times W_{\lambda}(p)\right) \cap\left(\mathbb{P}^{1} \backslash\{p\}\right) \times_{\mathbb{P}^{1}} \widetilde{W}_{\mu}$. For instance, consider a point $x \in \beta_{1}\left(\left(\mathrm{ev}_{1}^{p}\right)^{-1}\left(\mathfrak{X}_{\lambda}^{\prime} \cap \widetilde{\mathfrak{X}}_{\mu}^{\prime}\right)\right)$. Such a point corresponds to a degree $(d-1)$ map $\psi: \mathbb{P}^{1} \rightarrow L G$ with the resulting sheaf sequence modified at the point $p$. Let $\psi(p) \in L G$ correspond to the Lagrangian subspace $\Sigma$. The modification selects $\Sigma^{\prime} \subset \Sigma$ of codimension 1; this corresponds to a line $E \subset L G$. In the generic case, this line meets $\mathfrak{X}_{\lambda}$ in a unique point $r$ and $\widetilde{\mathfrak{X}}_{\mu}$ in a unique point $s$, both distinct from $\psi(p)$. The map from two $\mathbb{P}^{1}$ 's joined at a point to $\psi\left(\mathbb{P}^{1}\right) \cup E$ is a point of the space of stable maps; in fact, it is some

$$
\tilde{x} \in \bar{M}_{0,4}(L G, d) \times(L G \times L G)\left(\mathfrak{X}_{\lambda} \times \widetilde{\mathfrak{X}}_{\mu}\right),
$$

where we let marked points 1 and 2 land on $r$ and $s$, respectively, and take marked points 3 and 4 on the other component, distinct from $p$. By properness and transversality of a general translate, $\tilde{x}$ is the value at the special point of a family of such stable maps over Spec $\mathbb{C}[[t]]$, which is generically a smooth curve mapping to $L G$. Applying the morphism which forgets the first marked point, we get a $\mathbb{C}((t))$-valued point of $M_{0,3}(L G, d)=L M_{d}$ with limit point $x$ in the Quot scheme.

It remains only to show that the components indicated on the right-hand side of (46) are generically reduced. This is clear for the first-listed component, by transversality of a general translate. For the others, it is a linear algebra exercise to check, from the definition using rank conditions, that the scheme $W_{\lambda}(p) \cap \widetilde{W}_{\mu}(p)$ in the neighborhood of a point $\beta_{1}(x)$ for general $x \in\left(\operatorname{ev}_{1}^{p}\right)^{-1}\left(\mathfrak{X}_{\lambda}^{\prime} \cap \widetilde{\mathfrak{X}}_{\mu}^{\prime}\right)$ (resp. a point $\beta_{2}(x)$ for general $\left.x \in\left(\mathrm{ev}_{2}^{p}\right)^{-1}\left(\mathfrak{X}_{\lambda}^{\prime \prime} \cap \widetilde{\mathfrak{X}}_{\mu}^{\prime \prime}\right)\right)$ is contained in the image of the restriction of $\beta_{c}$ to $\pi_{c}^{-1}\left(\{p\} \times L M_{d-c}\right)$, where $c=1$ (resp. $c=2$ ). Recall, by Theorem 4 . that the restriction of $\beta_{c}$ is a locally closed immersion; now, the rank conditions, near $x$ on $\pi_{c}^{-1}\left(\{p\} \times L M_{d-c}\right)$, are the Schubert conditions in (37) or (38), and transversality of a general translate completes the argument.

This takes care of the assertions concerning cycles on $L Q_{d}$. A dimension count shows that every $k$-dimensional irreducible component in $\tau\left(p^{\prime \prime}\right)^{-1}\left(W_{\lambda}(p) \cap \widetilde{W}_{\mu}(p)\right)$ and in $\tau\left(p^{\prime \prime}\right)^{-1}\left(W_{\lambda}(p) \cap \widetilde{W}_{\mu}\left(p^{\prime}\right)\right)$ meets the open set where $\tau\left(p^{\prime \prime}\right)$ is an isomorphism. Now, exactly the same argument as above establishes the equality (45) and the case of (b) which concerns $L Q_{d}\left(p^{\prime \prime}\right)$.

We now establish the rational equivalences on $L Q_{d}$ - and on $L Q_{d}\left(p^{\prime \prime}\right)$ - which directly imply the quantum Giambelli formula of Theorem 1. 
Proposition 6. Fix $\lambda \in \mathcal{D}_{n}$ with $\ell=\ell(\lambda) \geqslant 3$. Set $r=2\lfloor(\ell+1) / 2\rfloor$. Let $p, p^{\prime}, p^{\prime \prime}$ denote distinct points in $\mathbb{P}^{1}$. Then we have the following identity of cycle classes

$$
\left[\left(\mathrm{ev}^{p}\right)^{-1}\left(\mathfrak{X}_{\lambda}\right)^{-}\right]=\sum_{j=1}^{r-1}(-1)^{j-1}\left[\left(\left(\mathrm{ev}^{p}\right)^{-1}\left(\mathfrak{X}_{\lambda_{j}, \lambda_{r}}\right) \cap\left(\mathrm{ev}^{p^{\prime}}\right)^{-1}\left(\widetilde{\mathfrak{X}}_{\lambda \backslash\left\{\lambda_{j}, \lambda_{r}\right\}}\right)\right)^{-}\right],
$$

both on $L Q_{d}$ and on $L Q_{d}\left(p^{\prime \prime}\right)$, where $\widetilde{\mathfrak{X}}_{\mu}$ denotes the translate of $\mathfrak{X}_{\mu}$ by a generally chosen element of the group $S p_{2 n}$.

Proof. Combining parts (a) and (b) of Proposition 5 gives

$$
\begin{aligned}
{\left[\left(\left(\mathrm{ev}^{p}\right)^{-1}\left(\mathfrak{X}_{\lambda_{j}, \lambda_{r}}\right) \cap\right.\right.} & \left.\left.\left(\mathrm{ev}^{p^{\prime}}\right)^{-1}\left(\widetilde{\mathfrak{X}}_{\lambda \backslash\left\{\lambda_{j}, \lambda_{r}\right\}}\right)\right)^{-}\right] \\
= & {\left[\left(\mathrm{ev}^{p}\right)^{-1}\left(\mathfrak{X}_{\lambda_{j}, \lambda_{r}} \cap \widetilde{\mathfrak{X}}_{\lambda \backslash\left\{\lambda_{j}, \lambda_{r}\right\}}\right)^{-}\right] } \\
& +\left[\beta_{1}\left(\left(\operatorname{ev}_{1}^{p}\right)^{-1}\left(\mathfrak{X}_{\lambda_{j}, \lambda_{r}}^{\prime} \cap \widetilde{\mathfrak{X}}_{\lambda \backslash\left\{\lambda_{j}, \lambda_{r}\right\}}^{\prime}\right)\right)^{-}\right] \\
& +\delta_{\ell, r}\left[\beta_{2}\left(\left(\operatorname{ev}_{2}^{p}\right)^{-1}\left(\mathfrak{X}_{\lambda_{j}, \lambda_{r}}^{\prime \prime} \cap \widetilde{\mathfrak{X}}_{\lambda \backslash\left\{\lambda_{j}, \lambda_{r}\right\}}^{\prime \prime}\right)\right)^{-}\right],
\end{aligned}
$$

for each $j$, with $1 \leqslant j \leqslant r-1$. Now (47) follows by summing and applying (i), (ii), and (in case $\ell=r$ ) (iii) of Corollary 5 .

The next result is equivalent to the quantum Giambelli relation (5).

Theorem 5. Suppose $\lambda \in \mathcal{D}_{n}$, with $\ell=\ell(\lambda) \geqslant 3$, and set $r=2\lfloor(\ell+1) / 2\rfloor$. Then we have the following identity in $Q H^{*}(L G)$ :

$$
\sigma_{\lambda}=\sum_{j=1}^{r-1}(-1)^{j-1} \sigma_{\lambda_{j}, \lambda_{r}} \sigma_{\lambda \backslash\left\{\lambda_{j}, \lambda_{r}\right\}}
$$

Proof. The classical component of (48) is the classical Pfaffian identity. Hence, (48) is equivalent to

$$
0=\sum_{j=1}^{r-1}(-1)^{j-1}\left\langle\sigma_{\lambda_{j}, \lambda_{r}}, \sigma_{\lambda \backslash\left\{\lambda_{j}, \lambda_{r}\right\}}, \sigma_{\mu}\right\rangle_{d}
$$

for every $d \geqslant 1$ and $\mu \in \mathcal{D}_{n}$ such that $|\lambda|+|\mu|=h(n, d)$. But (49) follows by applying the refined cap product operation $[\mathrm{F}, \S 8.1]$ along ev $\left(p^{\prime \prime}\right)$ to a general translate of $\mathfrak{X}_{\mu}$ in $L G$ and to both sides of (47) in $L Q_{d}\left(p^{\prime \prime}\right)$. On one side, we get zero, by Corollary 4 On the other side, we get the required alternating sum of Gromov-Witten numbers, by Corollary [3]

\section{Quantum Schubert calculus}

In this section, we use Theorem 1 and the algebra of $\widetilde{Q}$-polynomials to find explicit combinatorial rules that compute some of the quantum structure constants $e_{\lambda \mu}^{\nu}(n)$ that appear in the quantum product of two Schubert classes.

6.1. Algebraic background. The $\widetilde{Q}$-polynomials are the duals of certain modified Hall-Littlewood polynomials. More precisely, let $\left\{P_{\lambda}(X ; t)\right\}$ and $\left\{Q_{\lambda}(X ; t)\right\}$ be the usual Hall-Littlewood polynomials, defined as in [M] III.2]. Let $\left\{Q_{\lambda}^{\prime}(X ; t)\right\}$ be the adjoint basis to $\left\{P_{\lambda}(X ; t)\right\}$ for the standard scalar product on $\Lambda_{n}[t]$; we have $Q_{\lambda}^{\prime}(X ; t)=Q_{\lambda}(X /(1-t) ; t)$ in the notation of $\lambda$-rings (see [LLT]). According to [PR, Prop. 4.9], we have, for $\lambda \in \mathcal{E}_{n}$,

$$
\widetilde{Q}_{\lambda}(X)=\omega\left(Q_{\lambda}^{\prime}(X ;-1)\right)
$$


where $\omega$ is the duality involution of [M] I.2].

By property (b) of 2.1 there exist integers $e(\lambda, \mu ; \nu)$ such that

$$
\widetilde{Q}_{\lambda}(X) \widetilde{Q}_{\mu}(X)=\sum_{\nu} e(\lambda, \mu ; \nu) \widetilde{Q}_{\nu}(X) .
$$

The coefficients $e(\lambda, \mu ; \nu)$ are independent of $n$, and defined for any partitions $\lambda, \mu, \nu \in \mathcal{E}_{n}$. They occur in the second author's description of Schubert calculus for the Arakelov Chow ring of $L G$, considered as a scheme over the integers (see [T]).

There are explicit combinatorial rules (albeit, involving signs) for generating the numbers $e(\lambda, \mu ; \nu)$, which follow by specializing corresponding formulas for the multiplication of Hall-Littlewood polynomials (see [PR, Sect. 4] and [M] III.3.(3.8)]). In particular one has the following Pieri type formula for $\lambda$ strict ([PR Prop. 4.9]):

$$
\widetilde{Q}_{\lambda}(X) \widetilde{Q}_{k}(X)=\sum_{\mu} 2^{N(\lambda, \mu)} \widetilde{Q}_{\mu}(X)
$$

where the sum is over all partitions $\mu \supset \lambda$ with $|\mu|=|\lambda|+k$ such that $\mu / \lambda$ is a horizontal strip, and $N(\lambda, \mu)$ is the number of connected components of $\mu / \lambda$ not meeting the first column. Recall, by property (d) of 2.1 that

$$
\widetilde{Q}_{\lambda}(X) \widetilde{Q}_{n}(X)=\widetilde{Q}_{(n, \lambda)}(X),
$$

for any partition $\lambda \in \mathcal{E}_{n}$.

When $\lambda, \mu$ and $\nu$ are strict partitions, the $e(\lambda, \mu ; \nu)$ are classical structure constants for $L G(n, 2 n)$,

$$
\sigma_{\lambda} \sigma_{\mu}=\sum_{\nu \in \mathcal{D}_{n}} e(\lambda, \mu ; \nu) \sigma_{\nu},
$$

and hence are nonnegative integers. For strict $\lambda, \mu$ and $\nu$, Stembridge St has given a combinatorial rule for the numbers $e(\lambda, \mu ; \nu)$, analogous to the LittlewoodRichardson rule in type $A$. More precisely, we have

$$
e(\lambda, \mu ; \nu)=2^{\ell(\lambda)+\ell(\mu)-\ell(\nu)} f(\lambda, \mu ; \nu)
$$

where $f(\lambda, \mu ; \nu)$ is equal to the number of marked tableaux of weight $\lambda$ on the shifted skew shape $\mathcal{S}(\nu / \mu)$ satisfying certain conditions (for details, see loc. cit.). We also note that the $f(\lambda, \mu ; \nu)$ are the (classical) structure constants in the cup product decomposition

$$
\tau_{\lambda} \tau_{\mu}=\sum_{\nu \in \mathcal{D}_{n}} f(\lambda, \mu ; \nu) \tau_{\nu}
$$

where $\tau_{\lambda}, \tau_{\mu}$ and $\tau_{\nu}$ denote Schubert classes in the cohomology of the even orthogonal Grassmannian $O G(n+1,2 n+2)$ (see $[\mathrm{P}$, Sect. 6]).

Proposition 7. For $\lambda$ and $\nu$ in $\mathcal{D}_{n}$ and any integer $k$ with $1 \leqslant k \leqslant n$, we have

$$
e(\lambda, k ;(n+1, \nu))=2^{\ell(\lambda)-\ell(\nu)} e(\nu, n+1-k ; \lambda) .
$$

Proof. This follows from the combinatorial description of the structure constants given in (51). 
6.2. Quantum multiplication. Recall from the Introduction that for any $\lambda, \mu \in$ $\mathcal{D}_{n}$ there is a formula

$$
\sigma_{\lambda} \cdot \sigma_{\mu}=\sum e_{\lambda \mu}^{\nu}(n) \sigma_{\nu} q^{d}
$$

in $Q H^{*}(L G(n, 2 n))$. In Proposition 4 we showed that the quantum structure constants in degree $d=1$ for $L G(n, 2 n)$ are $1 / 2$ times classical structure constants for $L G(n+1,2 n+2)$. Using Theorem [1] the Pieri rule (51) and Proposition[7] we now get

Proposition 8 (Quantum Pieri Rule). For any $\lambda \in \mathcal{D}_{n}$ and $k \geqslant 0$ we have

$$
\sigma_{\lambda} \cdot \sigma_{k}=\sum_{\mu} 2^{N(\lambda, \mu)} \sigma_{\mu}+\sum_{\nu} 2^{N^{\prime}(\nu, \lambda)} \sigma_{\nu} q
$$

where the first sum is classical, as in (51), while the second is over all strict $\nu$ contained in $\lambda$ with $|\nu|=|\lambda|+k-n-1$ such that $\lambda / \nu$ is a horizontal strip, and $N^{\prime}(\nu, \lambda)$ is one less than the number of connected components of $\lambda / \nu$.

For any $d, n \geqslant 0$ and partition $\nu$, let $\left(n^{d}, \nu\right)$ denote the partition

$$
\left(n, n, \ldots, n, \nu_{1}, \nu_{2}, \ldots\right),
$$

where $n$ appears $d$ times before the first component $\nu_{1}$ of $\nu$. Theorem 1 now gives

Theorem 6. For any $d \geqslant 0$ and strict partitions $\lambda, \mu, \nu \in \mathcal{D}_{n}$ with $|\nu|=|\lambda|+|\mu|-$ $d(n+1)$, the quantum structure constant $e_{\lambda \mu}^{\nu}(n)$ (which is also the Gromov-Witten invariant $\left.\left\langle\sigma_{\lambda}, \sigma_{\mu}, \sigma_{\nu^{\prime}}\right\rangle_{d}\right)$ satisfies

$$
e_{\lambda \mu}^{\nu}(n)=2^{-d} e\left(\lambda, \mu ;\left((n+1)^{d}, \nu\right)\right) .
$$

Corollary 6. For any $d \geqslant 0$ and $\lambda, \mu, \nu \in \mathcal{D}_{n-1}$, the coefficient $e\left(\lambda, \mu ;\left(n^{d}, \nu\right)\right)$ is a nonnegative integer divisible by $2^{d}$.

Remark. It is not true that the coefficients $e(\lambda, \mu ; \nu)$ in (50) are all nonnegative. For example, we have

$$
\begin{aligned}
\widetilde{Q}_{3,2,1}\left(X_{4}\right) \cdot \widetilde{Q}_{3,2,1}\left(X_{4}\right)=8 & \widetilde{Q}_{4,4,4}+4 \widetilde{Q}_{4,3,2,2,1}+4 \widetilde{Q}_{4,2,2,2,2}-4 \widetilde{Q}_{4,4,2,2} \\
& +\widetilde{Q}_{3,3}\left(-4 e_{1} e_{2} e_{3}+2 e_{2}^{3}+4 e_{3}^{2}\right) \\
& +\widetilde{Q}_{1,1}\left(4 e_{1}^{2} e_{4}^{2}-4 e_{1} e_{2} e_{3} e_{4}+e_{2}^{2} e_{3}^{2}\right) .
\end{aligned}
$$

Example. It follows from the Remark that

$$
\sigma_{3,2,1} \cdot \sigma_{3,2,1}=q^{3}
$$

in $Q H^{*}(L G(3,6))$. This in turn implies that there is a single rational cubic curve passing through 3 general points on $L G(3,6)$. (See Corollary 8 for a generalization.)

6.3. Eight-fold symmetry. For any partition $\lambda \in \mathcal{D}_{n}$ with $\ell(\lambda)=r$, let $\lambda^{*}=$ $\left(n+1-\lambda_{r}, \ldots, n+1-\lambda_{1}\right)$. The next result illustrates a symmetry enjoyed by the Gromov-Witten invariants for $L G(n, 2 n)$.

Theorem 7. For any $d, e \geqslant 0$ with $d+e=\ell(\lambda)$, we have

$$
2^{n+d}\left\langle\sigma_{\lambda}, \sigma_{\mu}, \sigma_{\nu}\right\rangle_{d}=2^{\ell(\mu)+\ell(\nu)+e}\left\langle\sigma_{\lambda^{*}}, \sigma_{\mu^{\prime}}, \sigma_{\nu^{\prime}}\right\rangle_{e} .
$$

Also, for every $d>\ell(\lambda)$, we have $\left\langle\sigma_{\lambda}, \sigma_{\mu}, \sigma_{\nu}\right\rangle_{d}=0$. 
Proof. We can pass to real coefficients, and introduce

$$
\tilde{\sigma}_{\lambda}=2^{n / 4-\ell(\lambda) / 2} \sigma_{\lambda} \quad \text { and } \quad \widetilde{q}=q / 2 .
$$

Equation (54) is equivalent to the assertion that the coefficient of $\widetilde{\sigma}_{\nu^{\prime}} \widetilde{q}^{d}$ in $\widetilde{\sigma}_{\mu} \sigma_{\lambda}$ is equal to the coefficient of $\widetilde{\sigma}_{\mu} \widetilde{q}^{e}$ in $\widetilde{\sigma}_{\nu^{\prime}} \sigma_{\lambda^{*}}$. We observe (a restatement of quantum Pieri) that with respect to the basis $\left\{\widetilde{\sigma}_{\lambda}\right\}$ for $H^{*}(L Q, \mathbb{R})$, the matrices representing the operators

$$
-\cup \sigma_{i}: H^{2 *}(L G, \mathbb{R}) \rightarrow H^{2(*+i)}(L G, \mathbb{R})
$$

and

$$
\widetilde{q} \text {-coefficient }\left(-* \sigma_{n+1-i}\right): H^{2(*+i)}(L G, \mathbb{R}) \rightarrow H^{2 *}(L G, \mathbb{R})
$$

(where $*$ in (56) denotes the product in $Q H^{*}$ ) are transposes of each other. Using the quantum Giambelli formulas (4) and (5), we may express the operations $\widetilde{q}^{d}$-coefficient $\left(-* \sigma_{\lambda}\right)$ and $\widetilde{q}^{e}$-coefficient $\left(-* \sigma_{\lambda^{*}}\right)$ as expressions in (commuting) operators (55) and (56), and again they are transpose to each other. This establishes (54); the assertion that $\left\langle\sigma_{\lambda}, \sigma_{\mu}, \sigma_{\nu}\right\rangle_{d}=0$ when $d>\ell(\lambda)$ is also clear.

As a consequence of the previous results, we obtain some cases of a quantum Littlewood-Richardson rule in type $C$, specifically, expressions for $\sigma_{\lambda} \sigma_{\mu}$ whenever $\ell(\mu) \leqslant 3$.

Corollary 7. For any $\lambda \in \mathcal{D}_{n}$ and integers $a, b, c$ with $a>b>c$, we have

$$
\begin{aligned}
\sigma_{\lambda} \cdot \sigma_{a, b}=\sum_{\mu} e(\lambda,(a, b) ; \mu) \sigma_{\mu} & +\frac{1}{2} \sum_{\nu} e(\lambda,(a, b) ;(n+1, \nu)) \sigma_{\nu} q \\
& +\sum_{\rho} f\left(\rho,(a, b)^{*} ; \lambda\right) \sigma_{\rho} q^{2}, \\
\sigma_{\lambda} \cdot \sigma_{a, b, c}=\sum_{\mu} e(\lambda,(a, b, c) ; \mu) \sigma_{\mu} & +\frac{1}{2} \sum_{\nu} e(\lambda,(a, b, c) ;(n+1, \nu)) \sigma_{\nu} q \\
& +\sum_{\rho} f\left(\rho,(a, b, c)^{*} ;(n+1, \lambda)\right) \sigma_{\rho} q^{2} \\
& +\sum_{\eta} f\left(\eta,(a, b, c)^{*} ; \lambda\right) \sigma_{\eta} q^{3} .
\end{aligned}
$$

In particular, we see that all the quantum structure constants on the Lagrangian Grassmannians $L G(n, 2 n)$ with $n \leqslant 7$ are powers of 2 (prescribed by Theorem 7 and Proposition (4) times classical structure constants.

Note that in (57), we could have applied Theorem 7 to rewrite the second summand on the right as a structure constant involving $(a, b)^{*}$ :

$$
e(\lambda,(a, b) ;(n+1, \nu))=2^{\ell(\lambda)-\ell(\nu)} e\left(\nu,(a, b)^{*} ;(n+1, \lambda)\right) .
$$

The disappearance of the factors of 2 upon replacing the $e$ 's by $f$ 's after applying (54) is a more general phenomenon. For any partitions $\lambda, \mu$ and $\nu$, let us define the integer $f(\lambda, \mu ; \nu)$ using equation (52).

Proposition 9. For any $\lambda, \mu \in D_{n}$, we have

$$
\sigma_{\lambda} \sigma_{\mu}=\sum_{d+e=\ell(\mu)} \sum_{\nu \in \mathcal{D}_{n}} f\left(\nu, \mu^{*} ;\left((n+1)^{e}, \lambda\right)\right) \sigma_{\nu} q^{d}
$$


in $Q H^{*}(L G)$. In other words, any degree d quantum structure constant satisfies

$$
e_{\lambda \mu}^{\nu}(n)=f\left(\nu, \mu^{*} ;\left((n+1)^{e}, \lambda\right)\right)
$$

where $d+e=\ell(\mu)$.

Remarks. a) The identities (59) (which involves only classical structure constants) and (60) appear to be new. Note that (60) implies that any degree $d$ quantum structure constant $e_{\lambda \mu}^{\nu}(n)$, where $d=\ell(\mu)$ or $d=\ell(\mu)-1$, is equal to a classical structure constant.

b) For any $\lambda \in \mathcal{E}_{n}$ define the $\widetilde{P}$-polynomial $\widetilde{P}_{\lambda}(X)=2^{-\ell(\lambda)} \widetilde{Q}_{\lambda}(X)$; we have

$$
\widetilde{P}_{\lambda}(X) \widetilde{P}_{\mu}(X)=\sum_{\nu \in \mathcal{E}_{n}} f(\lambda, \mu ; \nu) \widetilde{P}_{\nu}(X) .
$$

In a companion paper to this one $\underline{\mathrm{KT} 2}$, we show that the multiplication of $\widetilde{P}$ polynomials describes the quantum cohomology ring of the spinor variety $O G(n+$ $1,2 n+2)$. At present we do not have a combinatorial interpretation for the coefficients $f\left(\lambda, \mu ;\left(n^{e}, \nu\right)\right)$ when $e \geqslant 2$.

If we fix an integer $d$ and strict partitions $\lambda, \mu, \nu$, then by repeated application of (54) we can relate 8 different Gromov-Witten invariants to each other. More precisely, if we define operators $A, B$ and $C$ by

$$
A\langle\lambda, \mu, \nu\rangle=\left\langle\lambda^{*}, \mu^{\prime}, \nu^{\prime}\right\rangle, \quad B\langle\lambda, \mu, \nu\rangle=\left\langle\lambda^{\prime}, \mu^{*}, \nu^{\prime}\right\rangle, \quad C\langle\lambda, \mu, \nu\rangle=\left\langle\lambda^{\prime}, \mu^{\prime}, \nu^{*}\right\rangle
$$

then $\{A, B, C\}$ generates a $(\mathbb{Z} / 2 \mathbb{Z})^{3}$-symmetry on the table of Gromov-Witten invariants. This symmetry dictates the vanishing of many of these numbers:

Proposition 10. Let $\lambda, \mu, \nu \in \mathcal{D}_{n}$ and let $d$ be an integer. The inequalities

$$
0 \leqslant d \leqslant \ell(\lambda) \text { and } \ell(\lambda)+\ell(\mu)-n \leqslant d \leqslant \ell(\lambda)+\ell(\mu)+\ell(\nu)-n
$$

are necessary conditions for the Gromov-Witten number $\left\langle\sigma_{\lambda}, \sigma_{\mu}, \sigma_{\nu}\right\rangle_{d}$ to be nonzero. Moreover, if the two sides of any of the inequalities in (61) differ by 0 or 1 , then $\left\langle\sigma_{\lambda}, \sigma_{\mu}, \sigma_{\nu}\right\rangle_{d}$ is related by the eight-fold symmetry to a classical structure constant.

As an application, we have the following rule for quantum multiplication by $\sigma_{\rho_{n}}$ :

Corollary 8. For any $\lambda \in \mathcal{D}_{n}$, we have $\sigma_{\lambda} \cdot \sigma_{\rho_{n}}=\sigma_{\lambda^{\prime *}} q^{\ell(\lambda)}$ in $Q H^{*}(L G(n, 2 n))$. In particular, $\sigma_{\rho_{n}} \cdot \sigma_{\rho_{n}}=q^{n}$.

\section{REFERENCES}

[AS] A. Astashkevich and V. Sadov : Quantum cohomology of partial flag manifolds $F_{n_{1}, \ldots, n_{k}}$, Comm. Math. Phys. 170 (1995), no. 3, 503-528.

[BGG] I. N. Bernstein, I. M. Gelfand and S. I. Gelfand : Schubert cells and cohomology of the spaces $G / P$, Russian Math. Surveys 28 (1973), no. 3, 1-26.

[Be] A. Bertram : Quantum Schubert calculus, Adv. Math. 128 (1997), no. 2, 289-305.

[BCF] A. Bertram, I. Ciocan-Fontanine and W. Fulton : Quantum multiplication of Schur polynomials, J. Algebra 219 (1999), no. 2, 728-746.

[Bo] A. Borel : Sur la cohomologie des espaces fibrés principaux et des espaces homogènes de groupes de Lie compacts, Ann. of Math. 57 (1953), 115-207.

[C] L. Chen : Quantum cohomology of flag manifolds, Adv. Math. 174 (2003), 1-34.

[C-F1] I. Ciocan-Fontanine : The quantum cohomology ring of flag varieties, Trans. Amer. Math. Soc. 351 (1999), no. 7, 2695-2729.

[C-F2] I. Ciocan-Fontanine : On quantum cohomology rings of partial flag varieties, Duke Math. J. 98 (1999), no. 3, 485-524. 
[D1] M. Demazure : Invariants symétriques des groupes de Weyl et torsion, Invent. Math. 21 (1973), 287-301.

[D2] M. Demazure: Désingularization des variétés de Schubert généralisées, Ann. Scuola Norm. Sup. Pisa Cl. Sci. (4) 7 (1974), 53-88.

[FGP] S. Fomin, S. Gelfand and A. Postnikov : Quantum Schubert polynomials, J. Amer. Math. Soc. 10 (1997), 565-596.

[F] W. Fulton : Intersection Theory, Second edition, Ergebnisse der Math. 2, Springer-Verlag, Berlin, 1998.

[FP] W. Fulton and R. Pandharipande : Notes on stable maps and quantum cohomology, in Algebraic Geometry (Santa Cruz, 1995), 45-96, Proc. Sympos. Pure Math. 62, Part 2, Amer. Math. Soc., Providence, 1997.

[FPr] W. Fulton and P. Pragacz: Schubert varieties and degeneracy loci, Lecture Notes in Math. 1689, Springer-Verlag, Berlin, 1998.

[GK] A. Givental and B. Kim : Quantum cohomology of flag manifolds and Toda lattices, Comm. Math. Phys. 168 (1995), no. 3, 609-641.

[G1] A. Grothendieck : Techniques de construction et théorèmes d'existence en géométrie algébrique IV: Les schémas de Hilbert, Séminaire Bourbaki 13 (1960/61), no. 221.

[G2] A. Grothendieck : Techniques de construction en géométrie analytique V: Fibrés vectoriels, fibrés projectifs, fibrés en drapeaux, in Familles d'espaces complexes et fondements de la géométrie analytique, Séminaire Henri Cartan 13 (1960/61), exposé 12.

[H] R. Hartshorne : Algebraic Geometry, Grad. Texts in Math. 52, Springer-Verlag, New York, 1977.

[HB] H. Hiller and B. Boe : Pieri formula for $S O_{2 n+1} / U_{n}$ and $S p_{n} / U_{n}$, Adv. in Math. 62 (1986), 49-67.

[KL] G. Kempf and D. Laksov : The determinantal formula of Schubert calculus, Acta Math. 132 (1974), 153-162.

[K1] B. Kim : Quantum cohomology of partial flag manifolds and a residue formula for their intersection pairings, Internat. Math. Res. Notices 1995, no. 1, 1-15.

[K2] B. Kim : On equivariant quantum cohomology, Internat. Math. Res. Notices 1996, no. 17, 841-851.

[K3] B. Kim : Quantum cohomology of flag manifolds $G / B$ and quantum Toda lattices, Annals of Math. 149 (1999), 129-148.

[KP] B. Kim and R. Pandharipande: The connectedness of the moduli space of maps to homogeneous spaces, preprint (2000), available at math.AG/0003168

[KM] M. Kontsevich, Y. Manin : Gromov-Witten classes, quantum cohomology, and enumerative geometry, Comm. Math. Phys. 164 (1994), no. 3, 525-562.

[KT1] A. Kresch and H. Tamvakis : Double Schubert polynomials and degeneracy loci for the classical groups, Ann. Inst. Fourier 52 (2002), no. 6, 1681-1727.

[KT2] A. Kresch and H. Tamvakis : Quantum cohomology of orthogonal Grassmannians, Compositio Math., to appear.

[LLT] A. Lascoux, B. Leclerc and J.-Y. Thibon : Fonctions de Hall-Littlewood et polynômes de Kostka-Foulkes aux racines de l'unité, C. R. Acad. Sci. Paris 316 (1993), 1-6.

[LT] J. Li and G. Tian : The quantum cohomology of homogeneous varieties, J. Algebraic Geom. 6 (1997), 269-305.

[LP] A. Lascoux and P. Pragacz : Operator calculus for $\widetilde{Q}$-polynomials and Schubert polynomials, Adv. Math. 140 (1998), no. 1, 1-43.

[M] I. G. Macdonald : Symmetric Functions and Hall Polynomials, Second edition, Clarendon Press, Oxford, 1995.

[P] P. Pragacz : Algebro-geometric applications of Schur $S$ - and Q-polynomials, Séminare d'Algèbre Dubreil-Malliavin 1989-1990, Lecture Notes in Math. 1478 (1991), 130-191, Springer-Verlag, Berlin, 1991.

[PR] P. Pragacz and J. Ratajski : Formulas for Lagrangian and orthogonal degeneracy loci; $\widetilde{Q}$-polynomial approach, Compositio Math. 107 (1997), no. 1, 11-87.

[R] J. Riordan : Combinatorial Identities, John Wiley \& Sons, New York, 1968.

[S] I. Schur : Über die Darstellung der symmetrischen und der alternierenden Gruppe durch gebrochene lineare Substitutionen, J. reine angew. Math. 139 (1911), 155-250.

[ST] B. Siebert and G. Tian : On quantum cohomology rings of Fano manifolds and a formula of Vafa and Intriligator, Asian J. Math. 1 (1997), no. 4, 679-695. 
[St] J. R. Stembridge : Shifted tableaux and the projective representations of symmetric groups, Adv. in Math. 74 (1989), 87-134.

[T] H. Tamvakis : Arakelov theory of the Lagrangian Grassmannian, J. reine angew. Math. 516 (1999), 207-223.

[Th] J. Thomsen : Irreducibility of $\bar{M}_{0, n}(G / P, \beta)$, Internat. J. Math. 9, no. 3 (1998), 367-376.

[V] C. Vafa : Topological mirrors and quantum rings, Essays on mirror manifolds, 96-119, Internat. Press, Hong Kong, 1992.

[W] E. Witten : The Verlinde algebra and the cohomology of the Grassmannian, Geometry, topology, \& physics, 357-422, Conf. Proc. Lecture Notes Geom. Topology, IV, Internat. Press, Cambridge, MA, 1995.

Department of Mathematics, University of Pennsylvania, 209 South 33rd Street, Philadelphia, PA 19104-6395

E-mail address: kresch@math.upenn.edu, harryt@math.upenn.edu 